\title{
Evolution and stability of the theoretically predicted families of periodic orbits in the $\mathrm{N}$-body ring problem
}

\author{
A. D. Pinotsis \\ Department of Astrophysics, Astronomy and Mechanics, Faculty of Physics, University of Athens, Panepistimiopolis, \\ 15784 Zographos, Athens, Greece \\ e-mail: apinots@phys.uoa.gr \\ Received 13 February 2004 / Accepted 7 September 2004

\begin{abstract}
We theoretically investigate the existence of families of periodic orbits in the planar $N$-body ring problem and we give a qualitative picture of the motion of a small particle. This study yields four families of periodic orbits which we also found numerically: two families of periodic orbits around the central body and two families around all the peripherals. These results are valid for different values of $N$ and $\beta$. Also we investigate the evolution of simple periodic motions as well as their stability. We found stable and unstable orbits around the central body, around all the peripherals and around one or more peripherals which form rings of stability. Some families present other types of bifurcations, such as bifurcations of families of non-symmetric periodic orbits of the same period and period-doubling bifurcations.
\end{abstract}

Key words. celestial mechanics - chaos

\section{Introduction}

Motions in a multi-body system constitute a difficult problem of considerable interest (Hadjidemetriou 1977, 1979; Meyer 1981; Palmore 1982). The dynamical study of multi-body systems can be simplified by using simpler $N$-body models based on the geometrical properties of the system (Markellos et al. 1997; Kalvouridis 1999a,b). We thus obtain valuable information on similar physical systems. Such a model is the coplanar $N$-body ring configuration, with $v=N-1$ spherical equimassive peripherals forming a regular polygon that rotates with constant angular velocity around a central primary, which has mass $\beta$ times larger than each of the peripherals.

Important information on such a dynamical system can be derived by studying its periodic orbits and their stability as known already by Poincaré (1899). When a periodic orbit of a family loses its stability for a value of the parameter $C$, another family bifurcates for the same value of $C$. Theoretical and numerical studies of bifurcations of families of periodic orbits in conservative Hamiltonian systems were provided first by Hénon (1965, 1970), Deprit \& Henrard (1968) and Contopoulos (1970, 2002).

In a previous work we investigated the $N=8$ body ring configuration, for $\beta=2$, and found symmetric periodic orbits (Pinotsis et al. 2002). In this paper, which is a continuation of our previous work, we theoretically ascertain four families of planar periodic motions in the $N$-body ring problem for different values of $N$ and $\beta$. We have also numerically found these families of simple and double symmetric periodic orbits and their stability for $N-1=4$ and 7 and for some values of $\beta$ (from 0.1 to 20). These families exist in the inner and outer region of the polygon surrounding the central body as well as the peripherals. Also, we investigate the evolution of some families of symmetric periodic orbits as well as the type of bifurcation in each case.

In this manner we have found both stable and unstable periodic orbits having different evolution as the energy changes. Some remain stable while others are always unstable and so form regions of stability. In some cases new families are generated by bifurcation.

The computation of some classes of orbits becomes more difficult as the number of peripherals becomes bigger. This is due to the increase of the collisions with the peripherals. This problem is a field of further study.

\section{Equations of motion}

A coplanar $N$-body ring configuration is made up of a central primary body of mass $M$ and $v$ peripherals of mass $m_{i}$ $(i=1,2, \ldots, v)$, where $m_{1}=m_{2}=\ldots=m_{v}$ (thus $\left.N=v+1\right)$ located at the vertices of a regular polygon. In order to have circular motion of the peripherals around the central primary with common constant angular velocity $\omega$, the gravitational forces must equal the centrifugal force so as to preserve the regularity of the polygon.

It is thus convenient to work in a synodic Cartesian coordinate system with the origin at the common mass centre of the ring configuration. The peripherals' coordinates in this system 
are (Kalvouridis 1999a)

$$
\begin{aligned}
& X_{i}=\frac{\alpha}{\sqrt{2(1-\cos \psi)}} \cos [(i-1) \psi], \\
& Y_{i}=\frac{\alpha}{\sqrt{2(1-\cos \psi)}} \sin [(i-1) \psi], \quad i=1,2, \ldots, v
\end{aligned}
$$

where $\alpha$ and $\psi$ are regular polygon constants, namely the distance between two successive peripherals and the angle $2 \pi / v$ respectively. For simplicity, a transformation of the physical coordinates to dimensionless ones is obtained through the relations

$x_{i}=\frac{X_{i}}{\alpha}, \quad y_{i}=\frac{Y_{i}}{\alpha}, \quad t=\omega \tau$

where $t, \tau$ is the time variable before and after the transformation respectively. Newton's second law of particle motion, the mass of which considered negligible, provides us with the following equations (Kalvouridis 1999a)

$\ddot{x}-2 \dot{y}=\frac{\partial U}{\partial x}, \quad \ddot{y}+2 \dot{x}=\frac{\partial U}{\partial y}$

where $U$ is the potential function. The latter equations are apparently similar to the ones of the restricted 3-body problem (Szebehely 1967).

The potential function $U$ is only spatially-dependent and is given by

$U=\frac{1}{2}\left(x^{2}+y^{2}\right)+\frac{1}{\Delta}\left(\frac{\beta}{r_{0}}+\sum_{i=1}^{v} \frac{1}{r_{i}}\right)$

where $\beta$ is the ratio $M / m_{i}$ and $\Delta$ is a constant determined by the geometrical properties of the polygon: $\Delta=K\left(\Lambda+\beta M^{2}\right)$, with $K=[2(1-\cos \psi)]^{1 / 2}$,

$\Lambda=\sum_{i=2}^{v} \frac{\sin ^{2} \theta \cos \left(\frac{v}{2}+1-i\right) \theta}{\sin ^{2}(v+1-i) \theta}$ and $\theta=\psi / 2$.

Also, $r_{0}, r_{i},(i=1,2, \ldots, v)$ are the dimensionless distances of the particle from the central primary and the peripherals respectively, with

$r_{0}=\sqrt{x^{2}+y^{2}}, r_{i}=\sqrt{\left(x-x_{i}\right)^{2}+\left(y-y_{i}\right)^{2}}, \quad i=1,2, \ldots, v$.

The Jacobian integral of Eqs. (3) is given by the following relation

$C=2 U-\left(\dot{x}^{2}+\dot{y}^{2}\right)$

where $C$ is the so-called Jacobian constant. Evidently, Eq. (6) is similar to that of the restricted 3-body problem (Szebehely 1967).

Our theoretical prediction of the existence of four families of planar periodic orbits around the central body and the peripherals is verified by numerical integration of the equations of motion (3), in double precision using a Runge-Kutta method of 7-8 order. Here, we use similar notations to our previous paper (Pinotsis 1987).

Of particular interest among the periodic orbits are the ones symmetric with respect to the rotating $x$-axis i.e. the axis joining the central primary with one peripheral (called peripheral 1). The numbering of the peripherals is counterclockwise. These start perpendicular of the $\mathrm{x}$-axis on the physical $(x-y)$ plane (initial conditions $x_{0} \neq 0, y_{0}=0, \dot{x}_{0}=0$, and $\dot{y}_{0} \neq 0$ ). Due to the symmetry of the periodic orbits, the stability indices are evaluated for half the orbit (Hénon 1970; Pinotsis 1987).

The stability of the periodic orbits can be studied by using the method of Hénon (1965) or by the evaluation of the monodromy matrix of the variational equations (Hadjidemetriou 1989). In this work the stability is studied by evaluation of the Hénon (1965) stability indices $a, b, c, d$, which are functions of the initial values $\left(x_{0}, \dot{x}_{0}\right)$ and the Jacobian $C$ (Hénon 1965). The orbits are stable if $|a+d| / 2<1$. The condition of symmetry gives $a=d$, making the stability condition $|a|<1$. The accuracy in the calculation of the coefficients $a, b, c, d$ is checked using their Jacobian determinant, which should be unity, i.e. $a d-b c=1$. Depending on the values of the stability coefficients we have six types of critical points for the symmetric periodic orbits. In the case when $a=+1$ and $c=0$ we have a critical point of type 1,2 or 3 . In type 1 , the main family of periodic orbits has a maximum or minimum $C$. In type 2 , the main family produces by bifurcation a family of symmetric periodic orbits of the same period. In type 3 , the main family produces by bifurcation a family of symmetric periodic orbits of the same period with a maximum or minimum $C$. Also, when $a=+1$ and $b=0$, we have a critical point of type $4,\left(\dot{x}_{0} \neq 0\right)$, and a new family of non-symmetric periodic orbits of the same period bifurcates at this point. In the case $a=-1$ we have a critical point where a bifurcation of double period family appears. This $1 \rightarrow 2$ transition point is of type 5 or 6 (Hénon $1965)$ if the coefficient $c=0\left(\dot{x}_{0}=0\right)$ or $b=0\left(\dot{x}_{0} \neq 0\right)$ correspondingly. Whenever we have a critical point of type 6 we change the $\mathrm{x}$-axis and so we study a critical point of type 5 .

\section{Theoretical prediction}

The function $U(x, y)=$ const. represents various regions of possible motion. We will give a qualitative picture of the motion of a small particle. Thus the following formulae have an approximate nature due to a number of assumptions. First consider periodic orbits far from the peripherals. Because of the symmetry of the problem, in the sidereal coordinate system the motion can be approximately considered as Keplerian, i.e. a central field motion. Hence, in the units used here the energy integral for an elliptical orbit with semi- major axis $a_{M}$ and eccentricity $e$ yields

$v_{e}^{2}=\frac{2}{r}-\frac{1}{a_{M}}$

where $v_{e}$ is the absolute value of the velocity vector since we study symmetric periodic orbits. For circular-like periodic orbits of radius $r$, Eq. (7) becomes

$v_{c}= \pm \frac{1}{\sqrt{r}}$

where the $(+)$ sign refers to the direct (counterclockwise) motion and the (-) to the retrograde motion, relative to the sidereal system. 
Also, considering the rotating (synodical) system, its velocity at a distance $r$ from the origin of the system is $v_{r}=r$ (in the units of our work where $\omega=1$ ) perpendicular to $r$. Thus in the synodic system the velocity of a circular-like orbit is

$v=v_{c}-r= \pm \frac{1}{\sqrt{r}}-r$.

As we can see from Eq. (9), four families of periodic orbits are possible. Due to the term $\pm 1 / \sqrt{r}$ we have two cases:

I) If $r>1$ outside the peripherals Eq. (9) yields:

- for direct motion in the fixed system the motion is retrograde in the rotating system;

- for retrograde motion in the sidereal system we also have retrograde motion in the synodical system.

Thus, in this case, we have two families of symmetric periodic orbits as in the case of the families 1 and $m$ of the restricted 3-body problem (Szebehely 1967). Eventually, a direct family with respect to the rotating system is not possible in this case, because the distance of each peripheral from the origin is (for $N-1=7) r_{\mathrm{p}}=1.1523824\left(r>r_{\mathrm{p}}>1\right)$. Also, the potential function becomes

$U=\frac{r^{2}}{2}+\frac{1}{r}$

and Eq. (6) gives then

$C=r^{2}+\frac{2}{r}-v^{2}$.

After taking into account Eq. (9), the last equation becomes

$C= \pm 2 \sqrt{r}+\frac{1}{r}$.

From Eq. (10) we can see that for $r \rightarrow \infty$ we have also $C \rightarrow \pm \infty$ (while the stability coefficient $a \rightarrow 1$ ). Equation (9) dominates more and more as the parameter $\beta$ increases.

II) If $r<1$, the orbits are inside the peripherals and surround the central body, since $r_{\mathrm{p}}>1>r$. In this case, Eq. (9) gives that:

- direct orbits in the fixed system are also direct in the rotating system;

- in the case of retrograde motion in the sidereal system we also have retrograde motion in the synodical system.

For increasing $C$ the periodic orbits shrink to the central body while the stability index $a$ tends to $1(C \rightarrow \infty, r \rightarrow 0, a \rightarrow 1)$.

These four families exist for different values of $N$ and $\beta$. We found these families of periodic orbits numerically, for $N-1=4,7$ and for different values of $\beta$ some of which are given below. We did not manage to find numerically any direct family of symmetric periodic orbits with respect to the rotating and the fixed system in the case $r>r_{\mathrm{p}}$. As $C$ decreases the periodic orbits of these four families approach the peripherals. After a characteristic value of $C$ for each family near the distance $r_{\mathrm{p}}\left(|r|>r_{\mathrm{p}}\right)$ these families evolve in the $(x, y)$ plane. We thus obtained different classes of periodic orbits among the peripherals, some of which we found numerically.

\section{Numerical results and discussion}

We found simple and double periodic orbits around the central primary, around all peripherals as well as orbits among the peripherals, and studied their stability for the cases $N-1=7,4$ and different values of $\beta$. Furthermore, a description of their evolution with respect to the Jacobian is given for some of them, focusing on bifurcation points. We found some types of bifurcations, namely period doubling pitchfork bifurcations of stable and unstable families as well as families of nonsymmetric periodic orbits of the same period. In further work we will study in detail all types of bifurcations.

The orbits found may be classified into four groups. This classification is based on the position of the orbits with respect to the peripherals as well as the central body. These groups include planetary-type orbits. For each of the above types of motion, diagrams of characteristics as well as stability curves are shown.

\subsection{Orbits around the central body}

First, we study the theoretically predicted families of simple symmetric periodic orbits, retrograde and direct, that surround the central body. These two families are generated from infinitesimal (circular) orbits around the central primary, as in the case of classes $\mathrm{f}$ or $\mathrm{h}$ and $\mathrm{g}$ or $\mathrm{i}$ of the restricted 3-body problem (Szebehely 1967). Thus, we can say that the beginning of the retrograde orbits family corresponds to Poincaré orbits of the first kind.

I) Sidereally and synodically retrograde orbits for $N-1=7$ and 4, family $I_{\mathrm{R}}$ :

Figures $1 \mathrm{a}$ and $1 \mathrm{~b}$ show a simple and a double periodic orbit for $N-1=7, \beta=10$ and $\beta=2$ on the $x-y$ plane with $C=1.95, x_{0}=-0.7083055, a=-0.615$ and $C=5.75$, $x_{0}=-0.1408290, a=1.064$ correspondingly. Also, Figs. $1 \mathrm{c}$ and $1 \mathrm{~d}$ show two simple periodic orbits for $N-1=4, \beta=1$ and $\beta=2$ on the $x-y$ plane with $C=2.44, x_{0}=-0.2985479$, $a=-0.503$, period $T=1.892$ and $C=1.72, x_{0}=-0.3384407$, $a=-0.932$, period $T=1.921$ correspondingly.

In Figs. $2 \mathrm{a}$ and $2 \mathrm{~b}$ the characteristic curve $x_{0}=x_{0}(C)$ and the respective stability curve $a=a(C)$ for the retrograde family in the case $N-1=7$ and $\beta=2$ is given. From Fig. 2a it is evident that when $C$ decreases, the orbits indeed move away from the central body, as the absolute value of their initial condition $x_{0}$ becomes larger and larger. For increasing $C, x_{0}$ tends to zero, that is, the orbits shrink to the central primary, while the stability index $a \rightarrow 1$. Thus for values of $C$ (Fig. 2b) $15<C$ the stability index is almost equal to 1 and so the orbits are "critically" stable for $C \rightarrow \infty$. For example, for $C=50$ it is $a=1.0000$, for $C=40$ it is $a=0.9999$. As $C$ decreases, the index $a$ also decreases, crossing the curve $a=0$ at $C=5.78$, and becomes negative.

Furthermore, the stability index becomes equal to $a=-1$ at $C=5.2061$, with $x_{0}=-0.4565275$ and $T=1.771(b=$ 0 and $c=0$ ). The stability curve at the bifurcation point is tangent to the straight line $a=-1$. This critical point is of type 5 and 6 simultaneously and we have there bifurcations of 

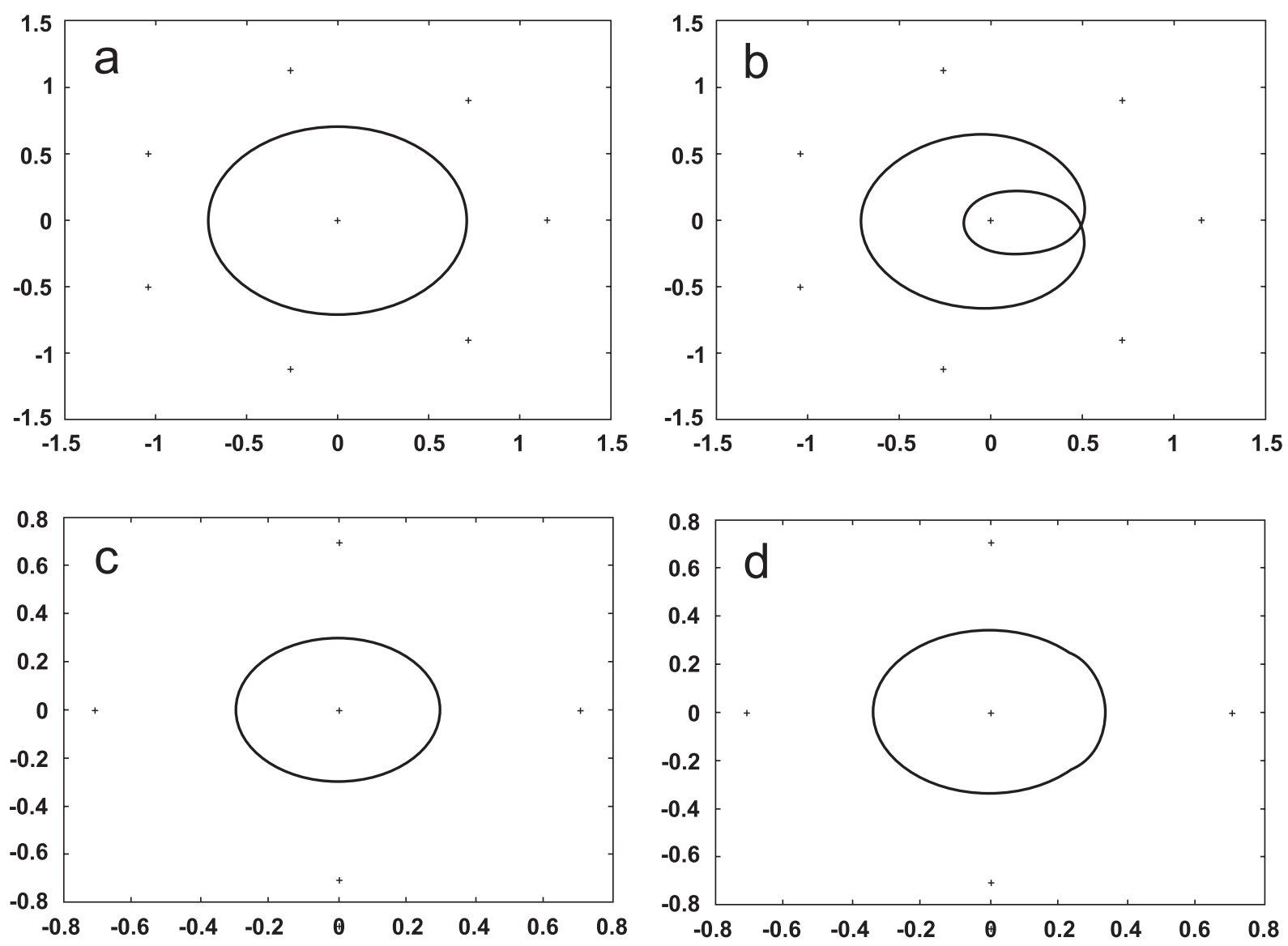

Fig. 1. a) Simple periodic orbit, $N-1=7, \beta=10$. b) Double periodic orbit, $N-1=7, \beta=2$. c) Simple periodic orbit, $N-1=4, \beta=1$. d) Simple periodic orbit, $N-1=4, \beta=2$.
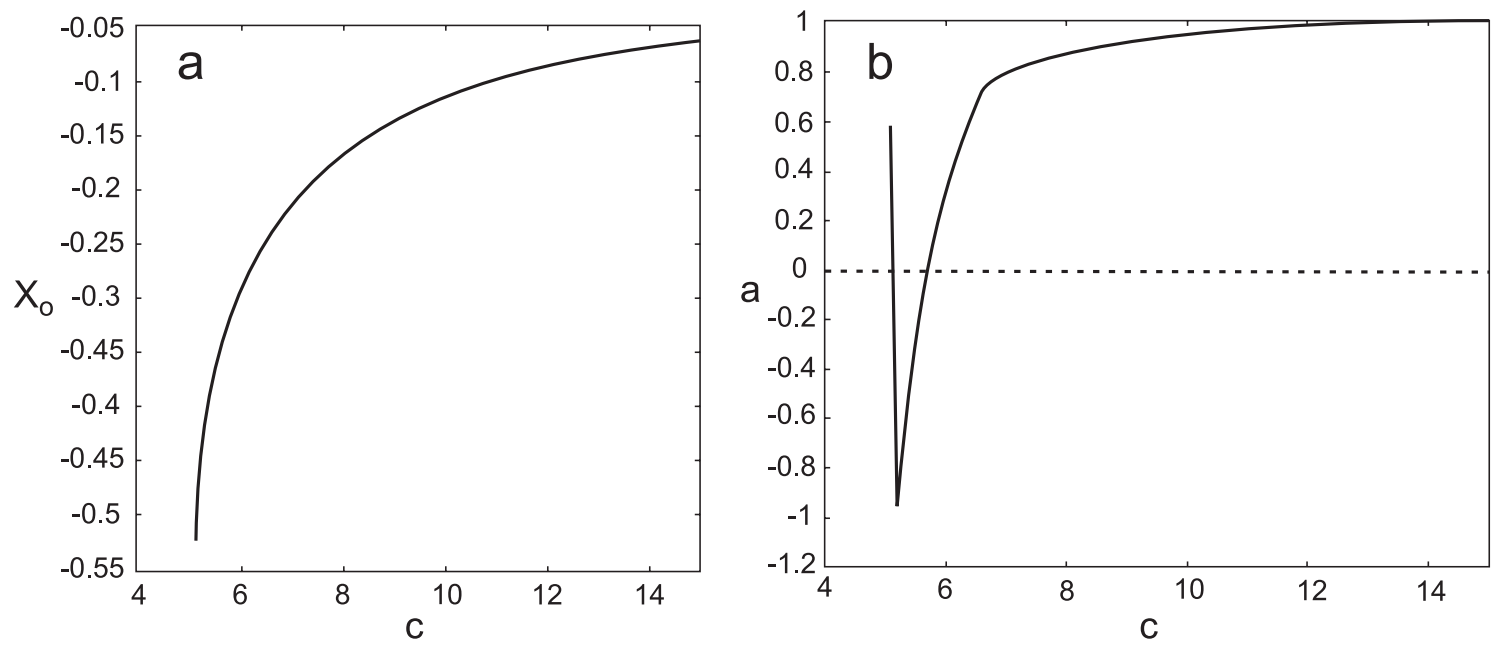

Fig. 2. a) Evolution of the $x-C$ curve (characteristic) of the $N-1=7, \beta=2$ class of periodic orbits. b) The stability curve of the same class of periodic orbits.

two new families of double periodic orbits with two branches each. The corresponding critical double periodic orbit belongs to three different families: to the family of simple symmetric periodic orbits and to two families $R_{c}, R_{b}$ of double periodic orbits that are symmetric to one another with respect to the origin.
After reaching the value $a=-1$, the stability index of the family $I_{\mathrm{R}}$ starts to increase as $C$ decreases until $a=0.914$ at $C=5.1357$. Consequently, as $C$ decreases this family remains stable and a ring of stability develops between $C_{2}=5.1357$, $x_{0}=-0.5263621$ and $C_{1}<\infty, x_{0}<0$ (we stopped the numerical calculations at $\left.C_{1}=50, x_{0}=-0.0158714\right)$. For a value 


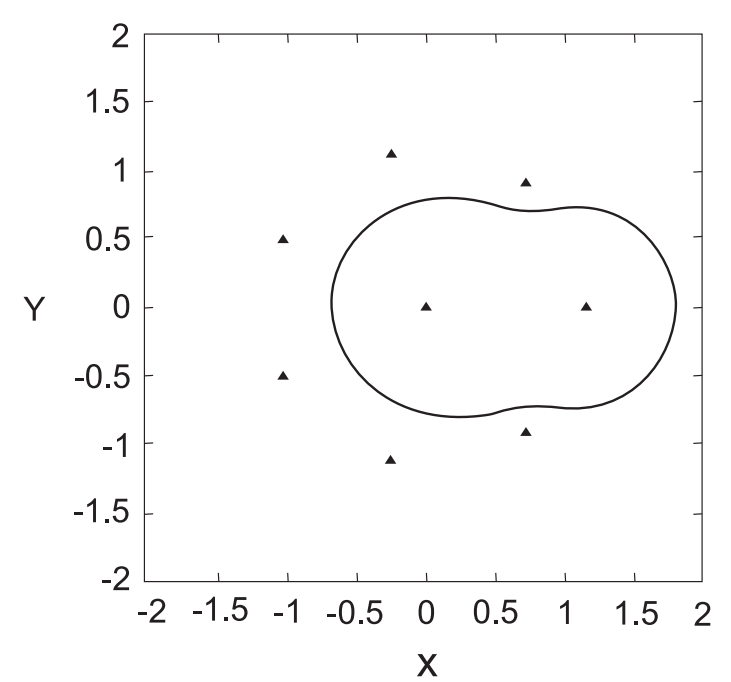

Fig. 3. Simple periodic orbit, $N-1=7, \beta=2$, for $C=5.12, x_{0}=$ $-0.6774304, a=3988, T=4.388$.

of $C$ a little smaller than 5.1357 a collision of the small body (particle motion) with the peripheral 1 appears.

The decrease of $C$ leads us to find different classes of periodic orbits among the peripherals as we mentioned in Sect. 3. For example, decreasing $C$ even more we found orbits surrounding both the peripheral 1 and the central body. In Fig. 3 we give an orbit of this type at $C=5.12, x_{0}=-0.6774304$ ( $a=3988$, period $T=4.388)$. This class evolves until a value of $C$ where there is a new collision of the small body with the peripheral 2 . Continuing to lower the values of $C$ we found orbits surrounding the central body and the peripherals 1,2 and $7\left(C=5.09, x_{0}=-0.7746856, a=7185\right.$, $T=6.491$ ); that is, orbits like the ones presented in Fig. 14a. If $C$ decreases further, another collision of the small body with peripheral 3 occurs and for even smaller values of $C$ orbits surrounding all the peripherals except 4 and 5 appear $(C=5.0$, $\left.x_{0}=-1.0308482, T=7.705, a=1117\right)$ and so on. This kind of orbits is presented in Fig. 15a.

II) Sidereally and synodically direct orbits for $N-1=7$ and 4 , family $I_{\mathrm{D}}$ :

In this case of the family of direct periodic orbits around the central primary, Figs. $4 \mathrm{a}$ and $4 \mathrm{~b}$ give a simple and a double periodic orbit for $N-1=7, \beta=10$ and $\beta=2$ on the $x-y$ plane with $C=5.44, x_{0}=-0.6919465, a=-0.623$ and $C=7.30648, x_{0}=-0.6056475, a=0.913$ respectively. Furthermore, Figs. $4 \mathrm{c}$ and $4 \mathrm{~d}$ show two simple periodic orbits for $N-1=4, \beta=10$ and $\beta=1$ on the $x-y$ plane with $C=2.0, x_{0}=-0.3480774, a=-0.921$ and $C=3.275$, $x_{0}=-0.2718221, a=4.631$ correspondingly.

The characteristic curve and the respective stability index diagrams in the case of the family of direct periodic orbits for $N-1=7$ and $\beta=2$ are presented in Figs. 5a and 5b. From the first curve, it is obvious that the orbits evolve in the same way as the retrograde ones. So, for $C$ decreasing they go far from the central body, while if $C$ increases they tend to shrink to it. The second one, the stability curve, shows a difference in the stability between the two families. Specifically, for values of $C$ (Fig. 5b) $12<C$, the stability index is almost equal to 1 , making the orbits critically stable for $C \rightarrow \infty$. Some indicative values are: for $C=50$ it is $a=1.000$, for $C=40$ it is $a=0.999$.

As $C$ decreases more the stability index also decreases until the value $a=0.213$ at $C=7.438, x_{0}=-0.3853219$. After this value the index $a$ starts to increase and becomes again equal to $a=1$ at the point $C=7.309, x_{0}=-0.4894866(b=0$ and $c=0$ ), so this point is a critical point of type 4 . At this point we have a bifurcation of a new family of non-symmetric periodic orbits of the same period. The stability curve is tangent to the straight line $a=1$.

After that for smaller $C$ the stability index decreases again as the Jacobian constant decreases, crosses the zero curve at $C=7.3025$ and becomes equal to $a=-1(b=0, c=0)$ at $C=7.30087$, with $x_{0}=-0.5253606$ and $T=6.442$. Thus, this point is a bifurcation point $1 \rightarrow 2$ and the corresponding double periodic orbit is of type 5 and 6 simultaneously. At the bifurcation point the stability curve is tangent to the straight line $a=-1$. This critical orbit belongs to three families: to the family of simple periodic orbits and to two families $D_{c}$ and $D_{b}$ of double symmetric periodic orbits, which contain periodic orbits symmetric to one another with respect to the origin. These two families are stable in the neighborhood of the critical point.

After this point, as $C$ decreases further, the stability index increases until $a=0.359(C=7.30064)$. Thus this family of direct simple periodic orbits is stable in the interval $C_{2}=$ 7.30064, $x_{0}=-0.5324479$ and $C_{1}<\infty, x_{0}<0$ (we stopped the numerical calculations at $\left.C_{1}=50, x_{0}=-0.0152669\right)$ forming a ring of stability. Finally, for $C$ smaller than $C=7.30064$ the orbits collide with a peripheral and so on. Thus a direct family is not possible to exist among the peripherals.

\subsection{Orbits around all the peripherals}

The two families existing in this case are retrograde only in the synodic system and surround all the peripherals as mentioned in Sect. 3. The first one is generated from approximately circular orbits, far from the peripherals for large positive values of $C$, while the other one is created also for circle-like periodic orbits far from the peripherals with the $C$ values being negative. As the absolute value of $C$ decreases, the periodic orbits of the two families approach the peripherals and get an elliptic shape, until a value of $C$ where there is a collision of the particle motion with a peripheral. When the absolute value of $C$ increases, the orbits move away from the peripherals.

I) Sidereally direct and synodically retrograde orbits for $N-1=7$ and 4, family $O_{\mathrm{DR}}$ :

In Figs. $6 \mathrm{a}$ and $6 \mathrm{~b}$ we give a simple and a double periodic orbit for $N-1=7, \beta=10$ and $\beta=2$ with $C=5.4, x_{0}=$ $-2.0033618, a=0.978$ and $C=7.33, x_{0}=-3.8748752, a=$ -1.078 correspondingly. Also, Figs. $6 \mathrm{c}$ and $6 \mathrm{~d}$ show two simple periodic orbits for $N-1=4, \beta=10$ and $\beta=2$ with $C=1.9$, $x_{0}=-1.3478185, a=13.38$ and $C=2.7, x_{0}=-1.6265422$, $a=-0.465$ correspondingly. 

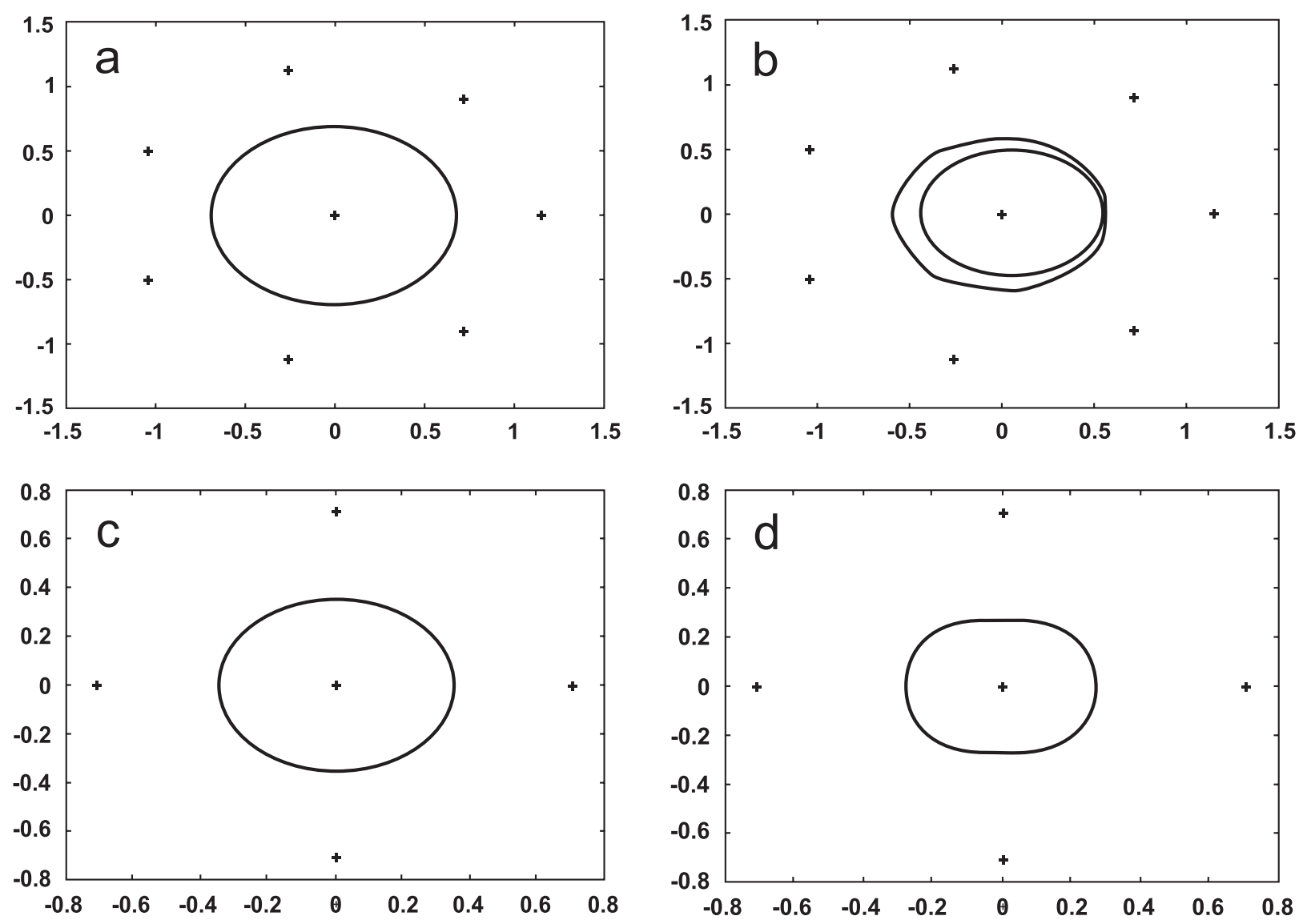

Fig. 4. a) Simple periodic orbit, $N-1=7, \beta=10$. b) Double periodic orbit, $N-1=7, \beta=2$. c) Simple periodic orbit, $N-1=4, \beta=10$. d) Simple periodic orbit, $N-1=4, \beta=1$.
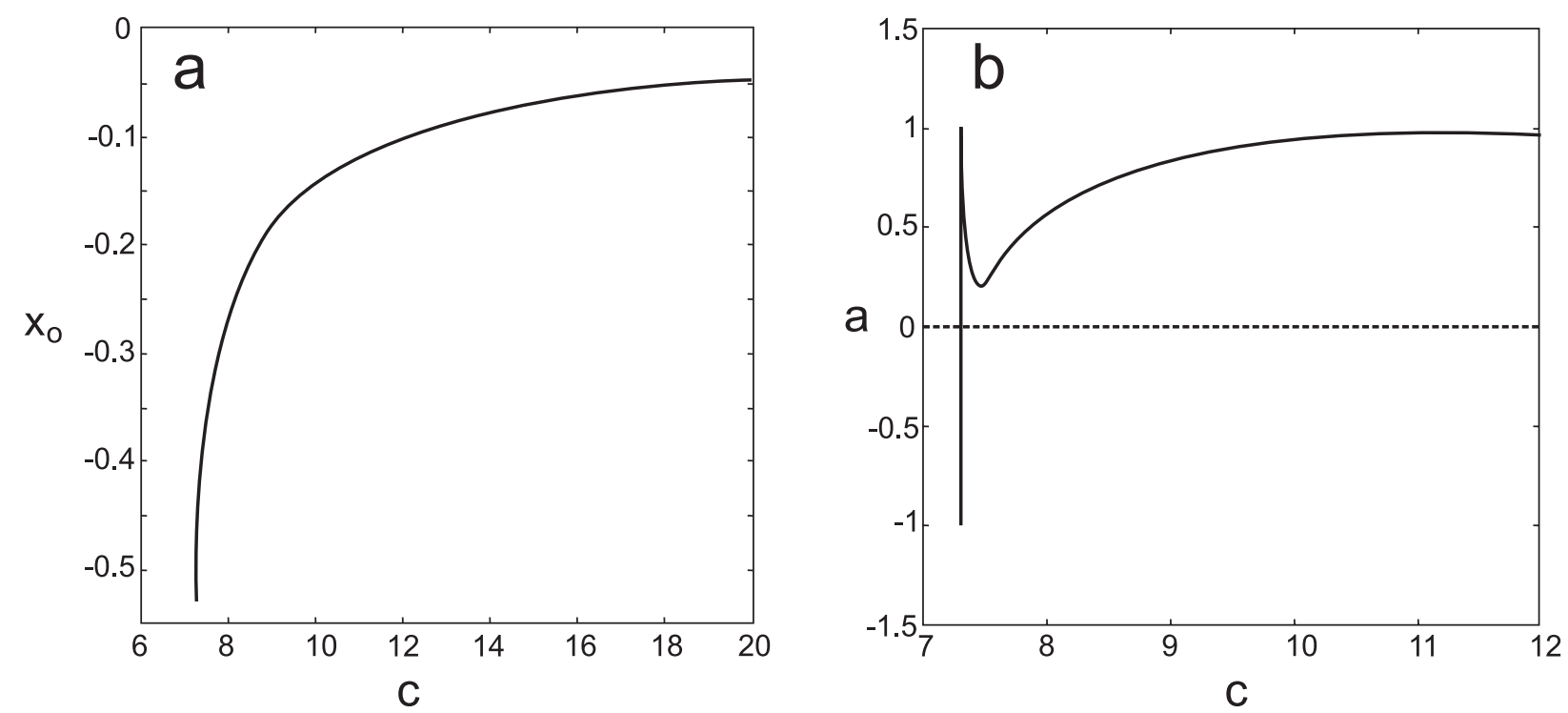

Fig. 5. a) Characteristic curve of the $N-1=7, \beta=2$ class of periodic orbits. b) Stability curve of the same class of periodic orbits.

In Fig. 7a we give the characteristic curve of the $C$-positive retrograde family for $N-1=7$ and $\beta=2$. Its stability curve is shown in Fig. 7b. From Fig. 7a it is obvious that when $C$ decreases, the orbits move near the ring configuration, while when $C$ increases the orbits move away the peripherals.
We found (Fig. 7b) that for $15<C$ the stability index $a$ takes values very close to 1 , thus the orbits tend to be critically stable. So, for $C \rightarrow \infty$ the absolute value of $x_{0} \rightarrow \infty$ while the coefficient $a \rightarrow 1$, as mentioned in Sect. 3. With $C$ decreasing, the stability index varies, remaining absolutely smaller than 1 

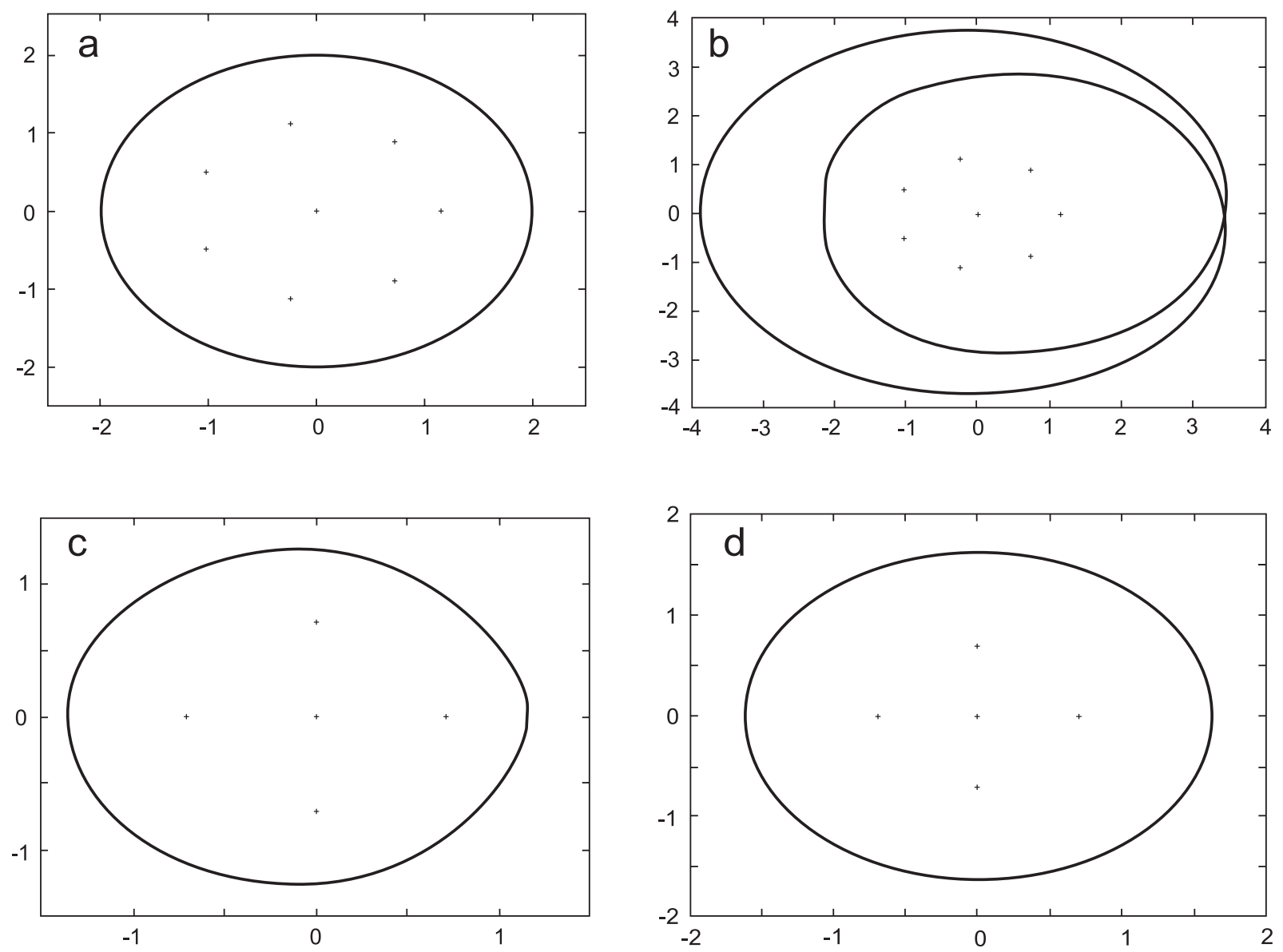

Fig. 6. a) Simple periodic orbit, $N-1=7, \beta=10$. b) Double periodic orbit, $N-1=7, \beta=2$. c) Simple periodic orbit, $N-1=4, \beta=10$. d) Simple periodic orbit, $N-1=4, \beta=2$.
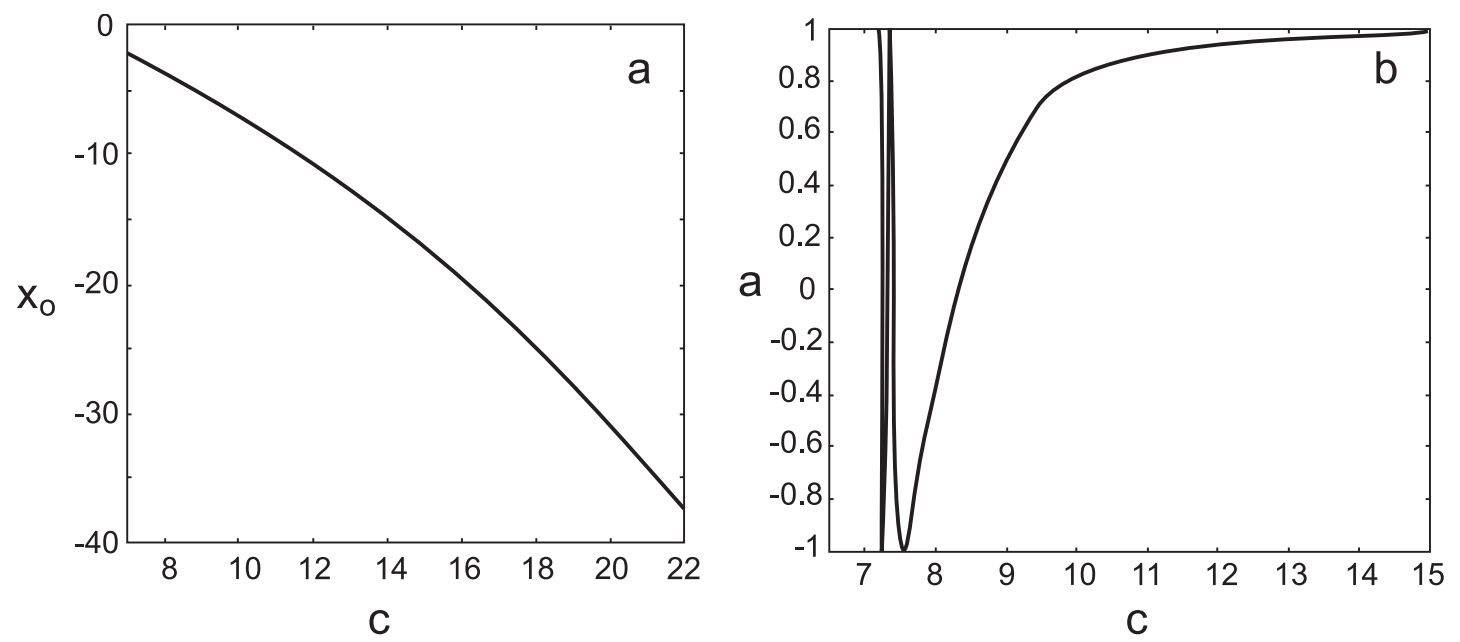

Fig. 7. a) Characteristic curve of the $N-1=7, \beta=2$ class of periodic orbits. b) Stability curve of the same class of periodic orbits.

and becomes equal to zero at $C=8.57$. It takes the value $a=-1$ at $C=7.531\left(x_{0}=-3.01017087, c=0, b=0\right.$, $T=9.904)$. At this $1 \rightarrow 2$ transition point, in which the stability curve is tangent to the straight line $a=-1$, a bifurcation of two new families with a double-period exists. Each bifurcating family of double symmetric periodic orbits, which has two branches in the form of a pitchfork, is stable in the region of the critical point. After this point, as $C$ decreases the stability index begins to increase and at $C=7.20\left(x_{0}=-2.2782601\right.$, $T=14.547$ ) we have $a=1$. At this critical point ( $b=0$ and $c=0$ ) a new family of non-symmetric simple periodic orbits bifurcates. 


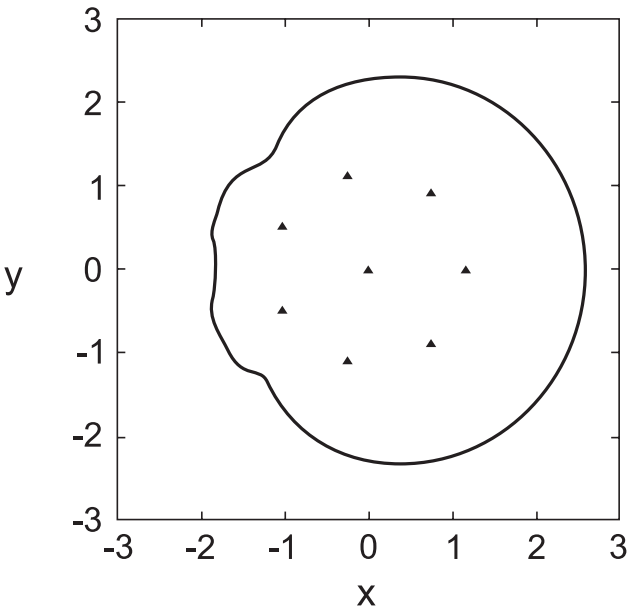

Fig. 8. Simple periodic orbit $(N-1=7, \beta=2)$, for $C=7.08, x_{0}=$ $-1.8362899, a=-32931, T=19.180$.

As $C$ decreases further the index $a$ continuously decreases and again takes the value $-1(b=0, c=0)$ at the point $C=7.1435, x_{0}=-2.0628003$. Thus we have a new $1 \rightarrow 2$ transition point. As $C$ continues to decrease the stability index increases (at $C=7.13, x_{0}=-2.0340109$ the index $a=1$ ) and takes very large values (of order $10^{4}$ ), but after a while decreases again to very small values (of order $-10^{4}$ at $C=7.103$ ). The orbit with initial condition $C=7.1, x_{0}=$ $-1.9016512(a=-3866, T=18.559)$ presents curves near the Lagrangian points, which evolve to two cusps at $C=7.08$, $x_{0}=-1.8362899(a=-32931, T=19.180)$. These cusps (Fig. 8) are located near the above Lagrangian points of equilibrium with coordinates $(-0.98, \pm 1.23)$ (Kalvouridis 1999a). The orbits near a Lagrangian point present a new dynamical feature compared to the remaining ones.

For even smaller values of $C$ the periodic orbits still surround all the peripherals presenting curves near their launch point at $C=7.05, x_{0}=-1.7437665$ and $a=-99.21$ $(T=13.083)$ until the small body collides with peripheral 3. After that, a new class of orbits arises; these orbits are inside the peripherals $3,4,5$ and 6 , presenting curves at these positions for $C=7.02, x_{0}=-1.7158825, a=-34172$ $(T=19.737)$ and becoming strongly unstable.

II) Sidereally and synodically retrograde orbits for $N-1=7$ and 4, family $O_{\mathrm{R}}$ :

In Fig. 9 we give two simple periodic orbits: a) for $N-1=$ 7, $\beta=2$ with $C=-4.4, x_{0}=-2.0792169, a=-0.075$; and b) for $N-1=4, \beta=2$ with $C=-2.5, x_{0}=-2.5779818$, $a=0.505$ correspondingly.

In Fig. 10a the characteristic curve of the family of orbits for negative $C(N-1=7, \beta=2)$ and in Fig. 10b the respective function $a(C)$ are shown. The former has a similar behaviour as the above $C$-positive family, though with different monotony: here when $C$ decreases the orbits move away from the ring, while when it increases the absolute value of $x_{0}$ decreases, thus the orbits move near the peripherals.

Figure 10b shows that the stability index never becomes -1 , and so a bifurcation of a double-period family does not exist. In particular, for $C$ less than -16 the stability index is very close to 1 and the orbits tend to be "critically" stable. For example, for $C=-20$ the stability index is $a=0.9988$, at $C=-30$ it is $a=0.9999$. Thus as we pointed out in Sect. 3, as $C \rightarrow-\infty$ the absolute value of $x_{0} \rightarrow \infty$ while the coefficient $a \rightarrow 1$. As $C$ grows, the absolute value of the stability index always remains less than 1 , becoming at first equal to zero at $C=-5.30$, and then $a=-0.144$ at $C=-4.63\left(x_{0}=-2.2907309\right.$, $T=4.018$ ). This value is a minimum as afterwards the stability index rises again to $a=0.075$ for $C=-4.3$. As $C$ increases further the stability index continuously increases and at the point $C=-4.20181, x_{0}=-1.7627857$ we have $a=1$ $(c=0)$, so the characteristic curve of the family has a maximum $C$. After this turning point, the family becomes more and more unstable as $C$ decreases while the absolute value of $x_{0}$ and the period of the orbits continuously decrease. At the point $C=-14.30, x_{0}=-1.1722734$ where the instability parameter is of the order of $10^{5}(T=1.546)$, the orbit is very close to all the peripherals. After this point there is a collision of the particle motion with one of the peripherals and the orbits, which are very unstable, differ from the ones before and so on. Thus we take various classes among the peripherals. For example, for the initial conditions $C=-5.0, x_{0}=-1.1170774(a=3505)$ and $C=-4.50, x_{0}=-0.7312398(a=1858)$ we take two orbits, one around the peripherals 4, 5 like the orbit I presented in Fig. 16a and another around the central body and the peripherals $1,2,7,3,6$ respectively. The last orbit is like the orbit I presented in Fig. 15a.

\subsection{Orbits around one peripheral}

In this case there also exist both retrograde and direct orbits, which are generated and evolve around one peripheral in the same way as the ones presented in the Sect. 4.1 and for this reason we will not give the figures of these orbits. In Fig. 11a the characteristic curve and in Fig. 11b the corresponding stability diagram for the family of retrograde periodic orbits around the peripheral $1(N-1=7, \beta=2)$ are given. From Fig. 11a it is obvious that as $C$ decreases, the orbits move away from the peripheral 1 , while for increasing $C$ the orbits move to it. Figure $11 \mathrm{~b}$ shows that for $C$ more than 12 the stability index is almost 1 , and so the orbits tend to be "critically" stable. For example, for $C=40\left(x_{0}=1.1421312\right)$ it is $a=1.0000$, while for $C=30$ ( $\left.x_{0}=1.1667548\right)$ it is $a=0.999$. As $C$ decreases, the stability index also decreases, crossing the curve $a=0$ at $C=6.26$, and at the point $C=5.88, x_{0}=0.8005645$ $(T=1.942)$ the stability index takes the value $a=-1(c=0)$. So, we have the existence of a double period bifurcation point of type 5 .

After the $1 \rightarrow 2$ bifurcation point the family of simple periodic orbits continues to be unstable as $C$ decreases. Its stability index reaches a minimum value about $a=-1.02$ and then increases and at the point $C=5.788, x_{0}=0.7592637$ becomes again $a=-1(b=0)$. At this $1 \rightarrow 2$ transition point of type 6 a new family of double symmetric periodic orbits with two branches bifurcates. This family is stable near the $1 \rightarrow 2$ transition point. As $C$ decreases the stability index 

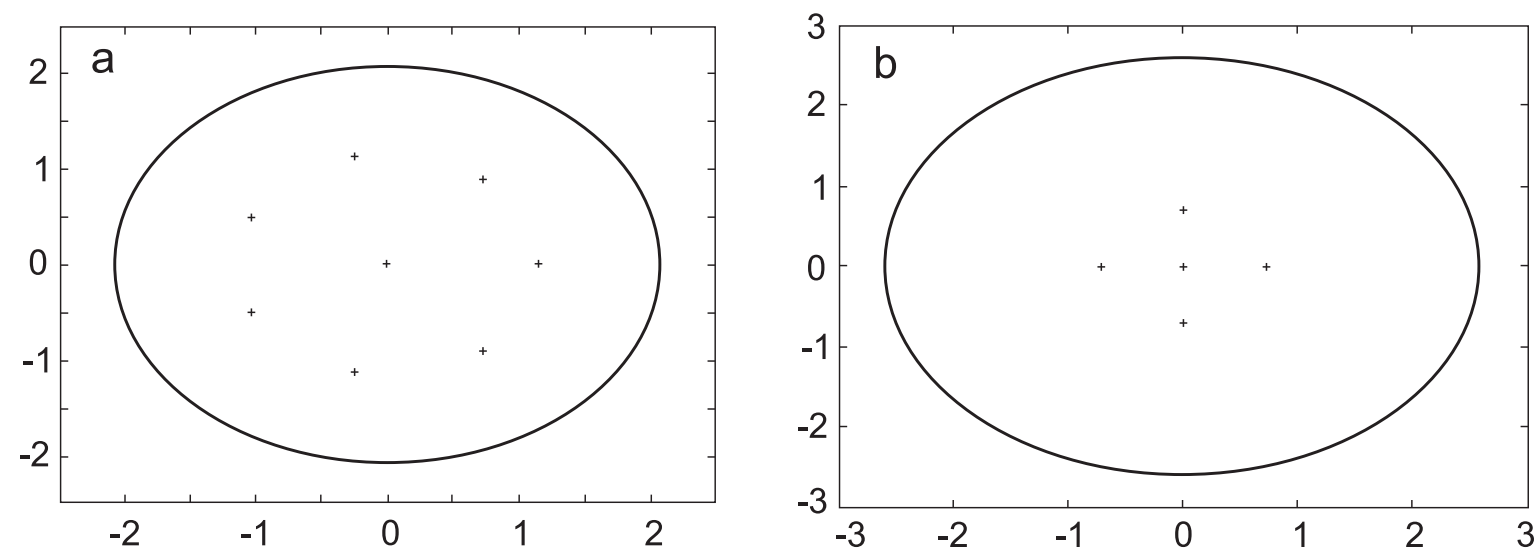

Fig. 9. a) Simple periodic orbit, $N-1=7, \beta=2$. b) Simple periodic orbit, $N-1=4, \beta=2$.
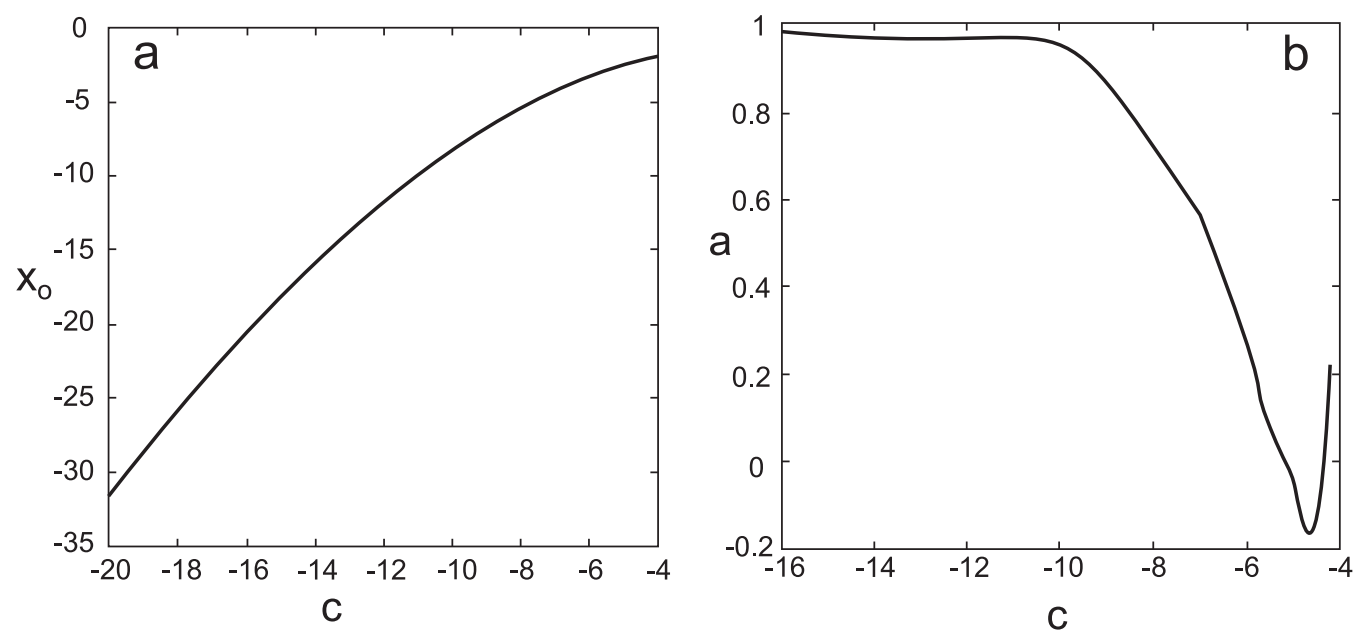

Fig. 10. a) Characteristic curve of the $N-1=7, \beta=2$ class of periodic orbits. b) Stability curve of the same class of periodic orbits.
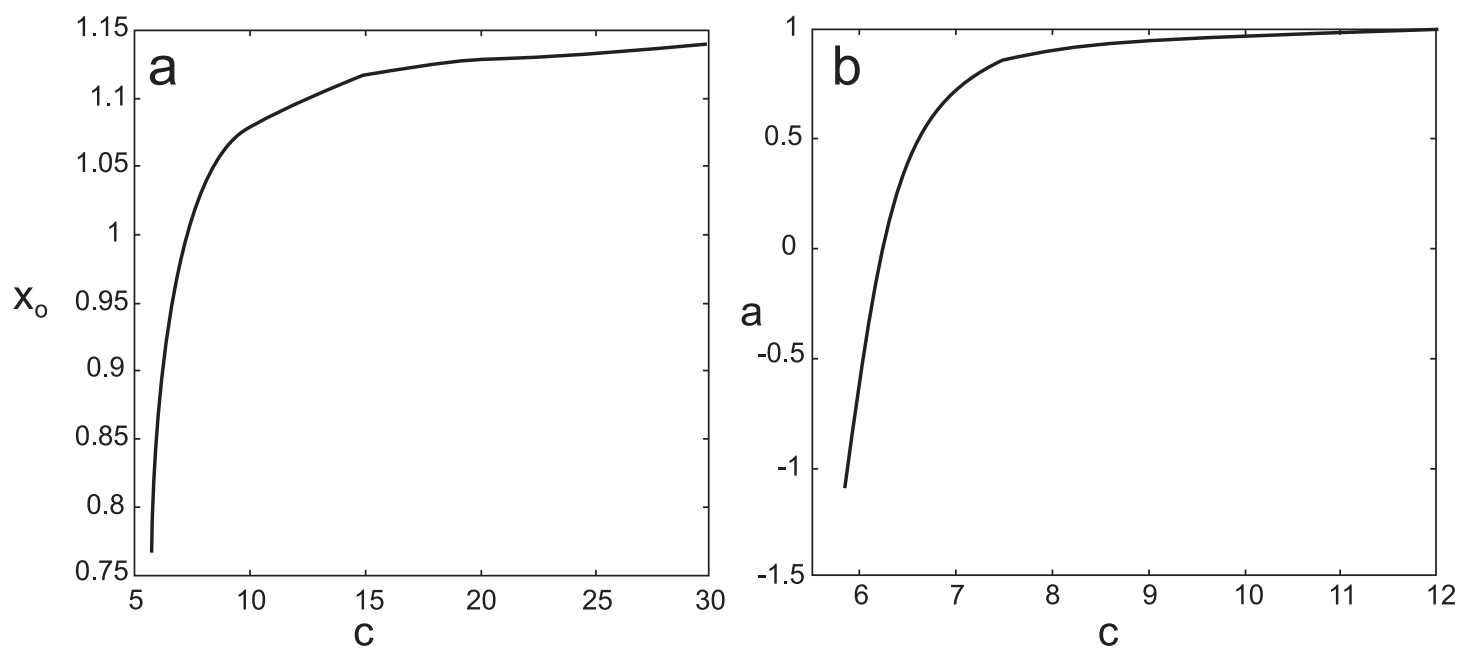

Fig. 11. a) Characteristic curve of the $N-1=7, \beta=2$ class of retrograde periodic orbits. b) Stability curve of the same class of periodic orbits.

continues to increase and at $C=5.7614$ and $x_{0}=0.7183741$ it is $a=1(c=0)$. Thus the characteristic curve has a minimum $C$. After this turning point as $C$ increases the value of $x_{0}$ decreases while the instability index increases further until a value of the order of $10^{3}$ at $C=6.60, x_{0}=0.5509206$. After that a collision of the particle motion with one of the neighbouring peripherals arises and the orbits start to surround more than one peripheral. 

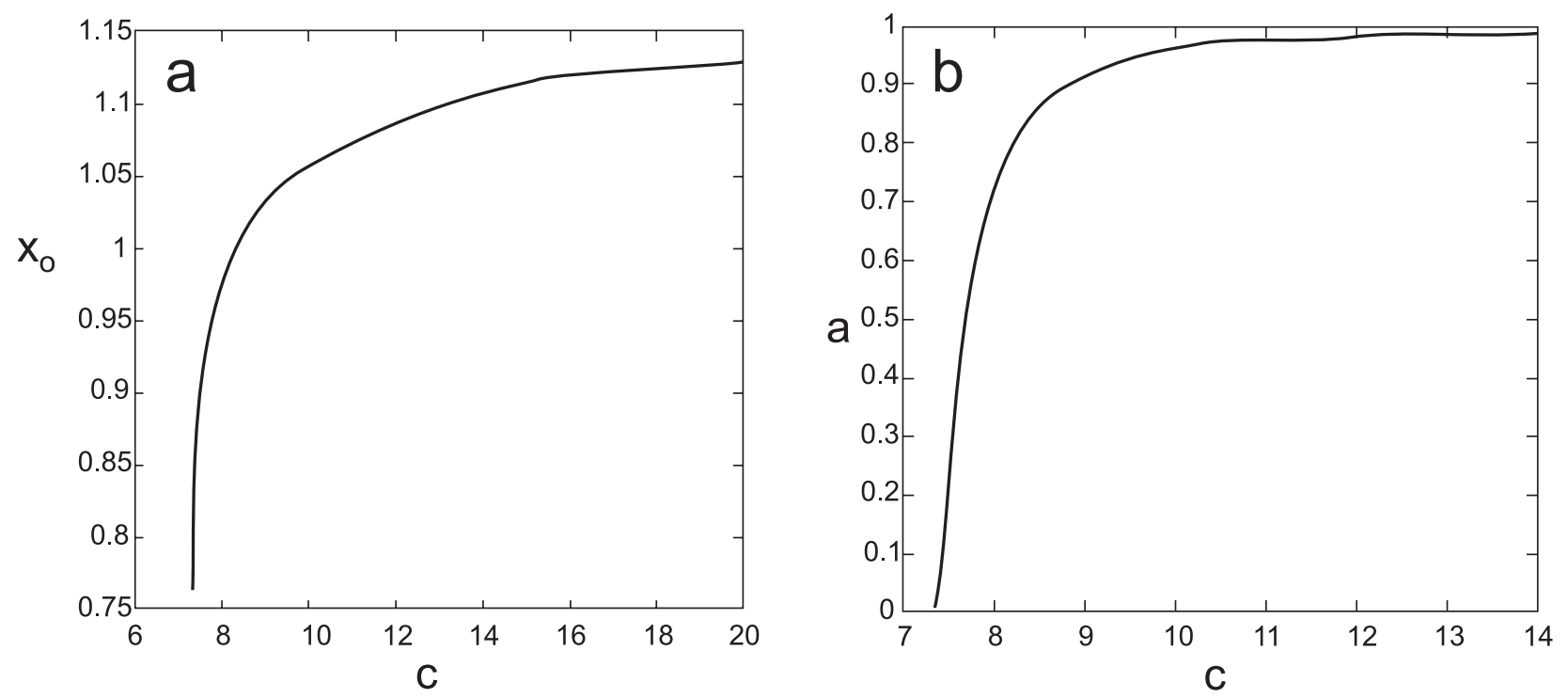

Fig. 12. a) Characteristic curve of the $N-1=7, \beta=2$ class of direct periodic orbits. b) Stability curve of the same class of periodic orbits.

As far as the direct family is concerned, in Fig. 12a we have the characteristic and in Fig. 12b the respective stability curve. From the first of these we conclude that the evolution of the family with $C$ is similar to that of the retrograde orbits: when $C$ decreases the orbits go far from the peripheral 1, while when $C$ increases they tend to approach it, covering less and less area in the plane.

The stability diagram of this family shows that for $C$ greater than 14 the stability index is almost equal to 1 . As $C$ continues to decrease the stability index decreases and at the point $C=7.3352, x_{0}=0.7272765$ the parameter $a=-1(b=0)$. This point is a $1 \rightarrow 2$ bifurcation point of type 6 where a family of double periodic orbits bifurcates. As $C$ decreases further the instability of the family increases and reaches the value $a=-234$ at $C=7.31, x_{0}=0.7117237$. Then the orbit collides with one of the neighbouring peripherals.

Due to the symmetry of the problem, orbits of this type exist around each one of the 7 peripherals. The behaviour and evolution of these orbits is similar to the previous ones, but the new classes arising after a collision of the small body differ according to the respective peripheral. This is due to the fact that this type of motion is not symmetric to the $x$-axis of the synodical system but to the $x$-axis of the local system; that is, the parallel axis to the synodic $x$-axis, passing from the respective peripheral. We give the initial conditions of a retrograde periodic orbit surrounding the peripheral 2 for $C=7.0, x_{0}=0.5446427$ ( $T=0.683)$ and stability coefficient $a=0.725$. Another orbit there is for $C=6.2, x_{0}=0.4623543,(T=1.192)$ and $a=-0.996$. Also, an orbit around the peripheral 3 is $C=7.0$, $x_{0}=-0.429456(T=0.682)$ and $a=0.734$.

\subsection{Orbits among the peripherals}

We mentioned in previous sections that the evolution of the theoretically predicted families yields various classes of symmetric periodic orbits. These orbits curve and twist among the

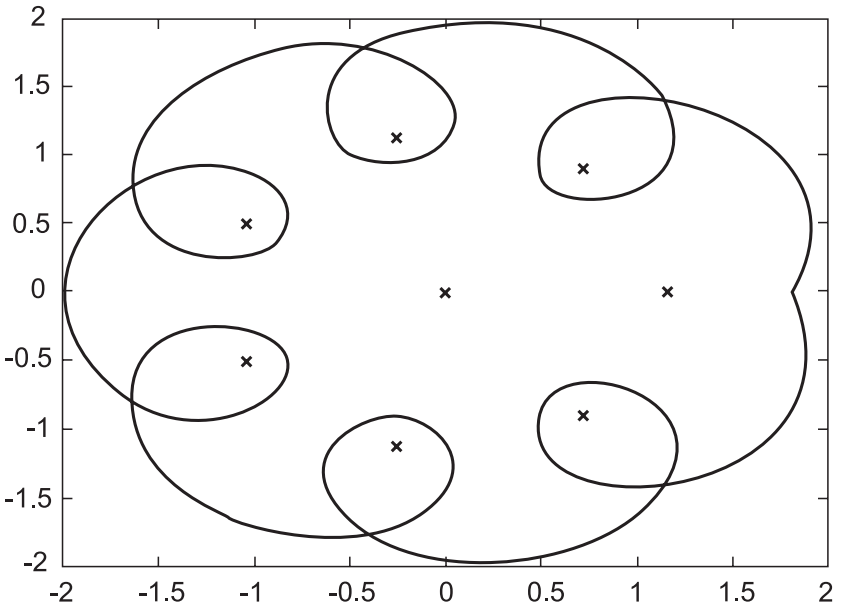

Fig. 13. Simple periodic orbit, $N-1=7, \beta=2$ : for $C=5.4, x_{0}=$ $-1.9958255, a=23746, T=18.156$.

peripherals, surrounding two or more of them. For example, Fig. 13 shows an orbit which has 6 loops around the symmetric peripherals $2,7,3,6,4,5$ and surrounds the central body as well as the peripheral 1 . This orbit results from the retrograde C-positive periodic orbits surrounding all the peripherals. We will give some of these periodic orbits (for $N-1=7, \beta=2$ ) as well as the previously mentioned orbits $14 \mathrm{a}$ and $15 \mathrm{a}$ (Sect. 4.1 case I) which result from the retrograde periodic orbits around the central body. Also, as it is mentioned in Sect. 4.2 case II, orbits like the orbit I (with C-negative) presented in Fig. 15a may result from orbits of the family $O_{\mathrm{R}}$.

In Figs. 14b and 14c we give the characteristic and the stability curves of the new class of periodic orbits which surround the central body as well as the peripherals 1,2 and 7 (Fig. 14a). Also, Figs. 15b and 15c show the characteristic and the stability curves respectively for the other new class the orbits of which surround all the peripherals except the symmetric ones 4 

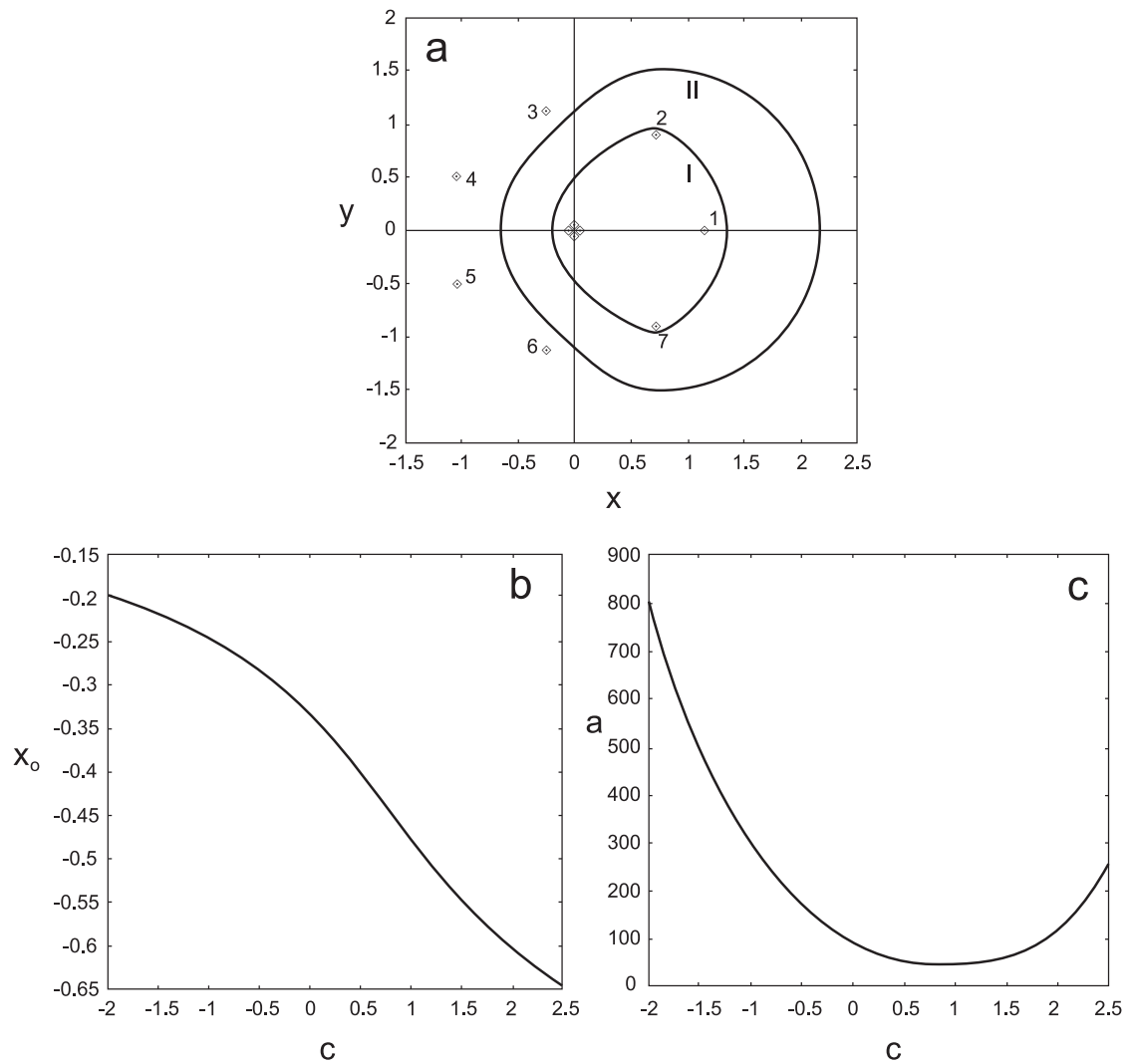

Fig. 14. a) Two simple periodic orbits, $N-1=7, \beta=2$ : for (I) $C=-2.1, x_{0}=-0.1910466, a=885, T=1.587$ and (II) $C=2.70$, $x_{0}=-0.6618065, a=355, T=4.188$. b) Characteristic curve of the above class of the periodic orbits. c) Stability curve of the above class of periodic orbits.
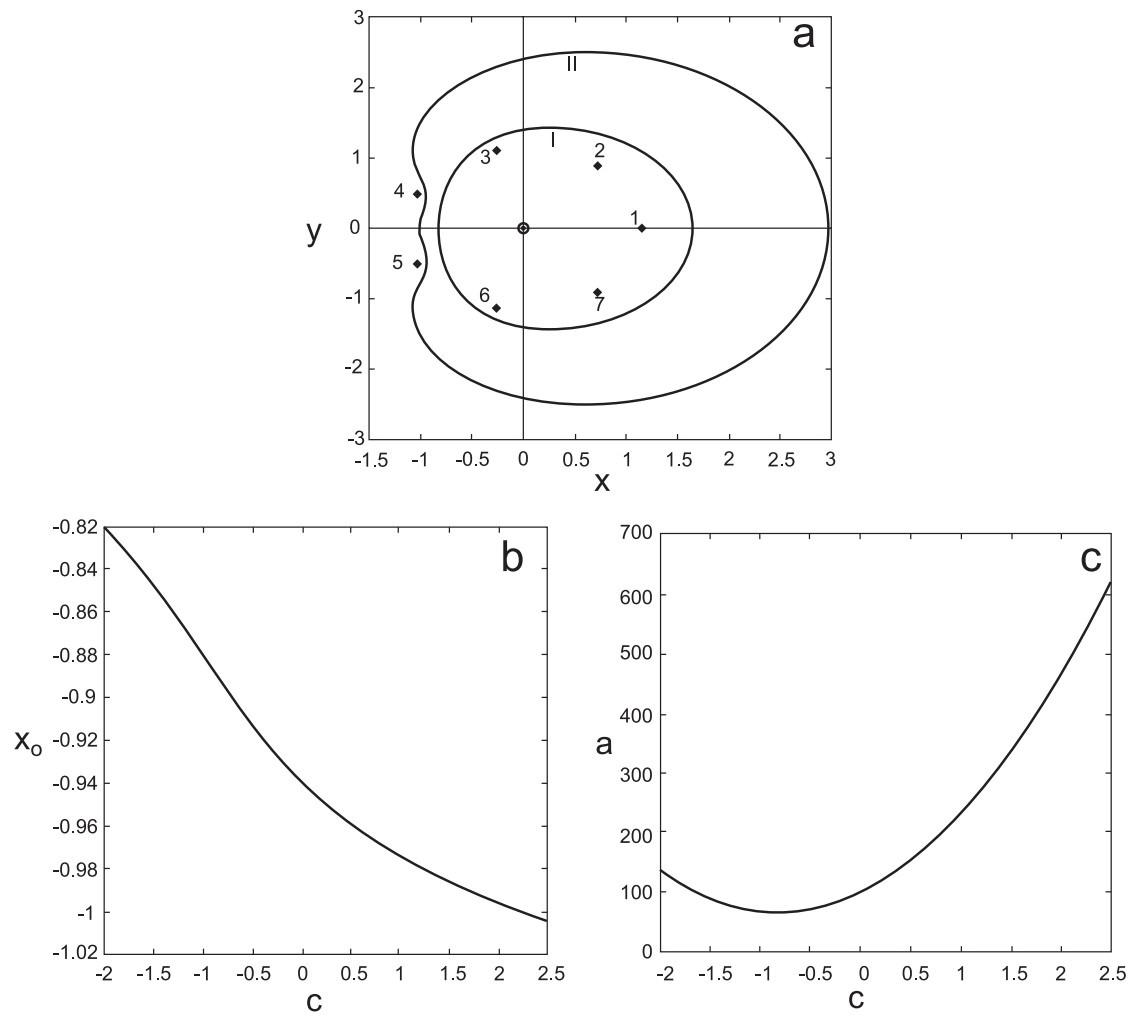

Fig. 15. a) Two simple periodic orbits, $N-1=7, \beta=2$ : for (I) $C=-2.1, x_{0}=-0.8153107, a=156, T=2.699$ and (II) $C=5.0$, $x_{0}=-1.0308482, a=1117, T=7.696$. b) Characteristic curve of the above class of the periodic orbits. c) Stability curve of the above class of periodic orbits. 

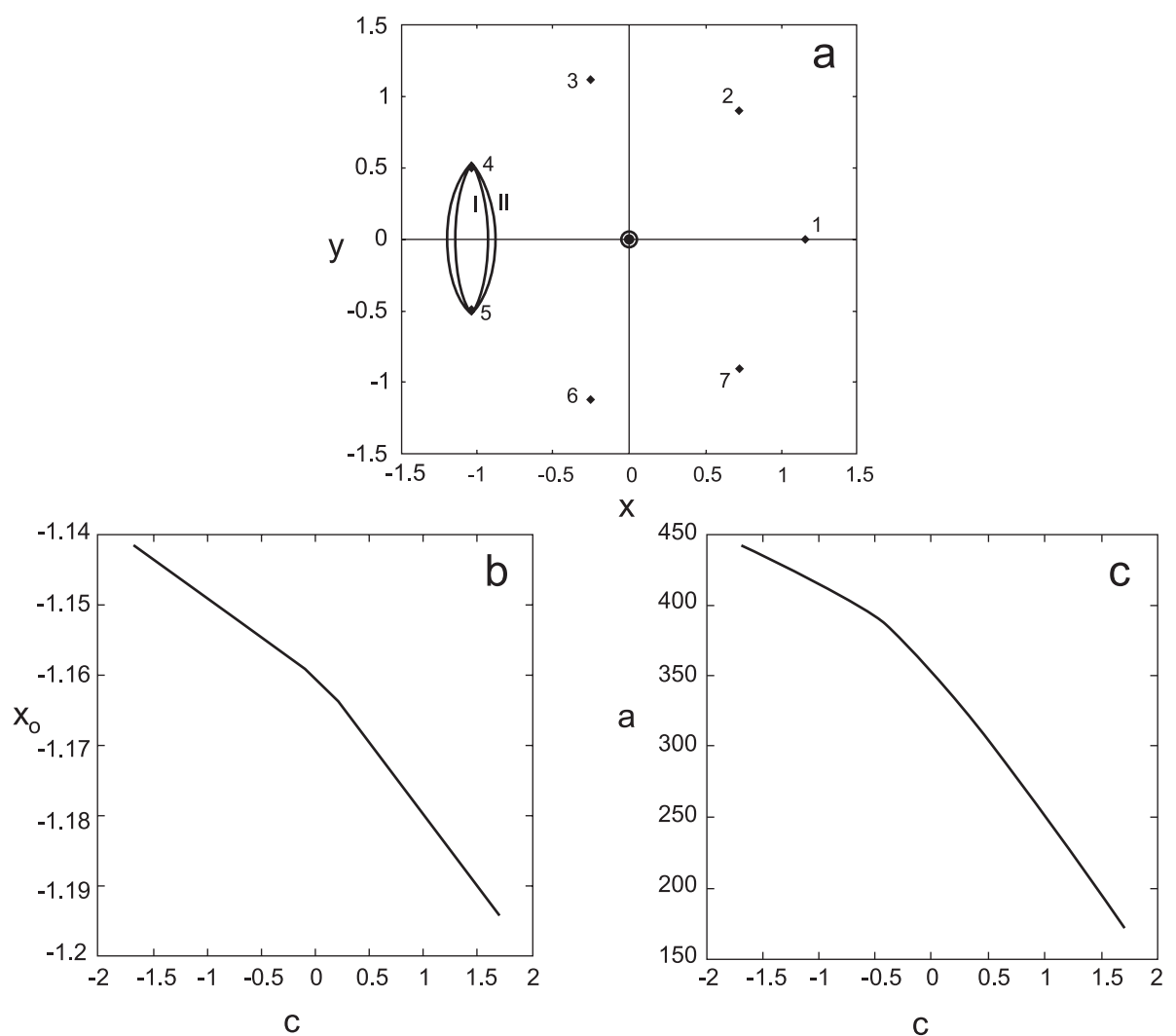

Fig. 16. a) Two simple periodic orbits, $N-1=7, \beta=2$ : for (I) $C=-1.9, x_{0}=-1.1406088, a=718, T=0.598$ and (II) $C=1.9$, $x_{0}=-1.2005553, a=155, T=0.799$. b) Characteristic curve of the above class of the periodic orbits. c) Stability curve of the above class of periodic orbits.

and 5 (Fig. 15a). These two classes are very unstable and appear a minimum of the stability index as $C$ changes, $a=43.89$ at $C=0.97, x_{0}=-0.4709292$ and $a=65.37$ at $C=-0.87$, $x_{0}=-0.8889418$ correspondingly. The orbits move more and more towards the internal or the external peripherals as $C$ decreases or increases.

Further development of our calculations is associated with the unstable character of new classes of retrograde symmetric periodic orbits. For example, in Fig. 16a we give two orbits surrounding the peripherals 4 and 5. As mentioned in Sect. 4.2 case II, orbits like the orbit I $(C=-1.90)$ may result from retrograde periodic orbits around all the peripherals, while orbits like the orbit II $(C=1.90)$ may result from the retrograde periodic orbits around the central body if the initial condition $x_{0}$ is far enough. As it is mentioned in Sect. 4.3 such orbits (nonsymmetric periodic orbits with respect to the rotating $x$-axis) may also result from the retrograde periodic orbits around the peripheral 4 or 5. In Figs. 16b and 16c we show the characteristic and the stability curves correspondingly. This family is very unstable. As $C$ decreases, the orbits approach more and more the two peripherals and for a certain value of $C$ a collision appears with one of them.

When $C$ increases such that $1.90<C$ (Fig. 16a) and after a collision with the central body, there will be periodic orbits surrounding it. Thus, we found a new class of retrograde periodic orbits around the central body and the symmetric peripherals 4 and 5 (Fig. 17a). In Figs. 17b and 17c the characteristic and the respective function $\mathrm{a}(C)$ of this class are presented. As we can see the class is very unstable and the stability index has a minimum $a=30.78$ at $C=2.0, x_{0}=-1.4579073$. As $C$ decreases further the orbits approach more the internal peripherals, while moving towards the external ones as $C$ increases.

In the same way we found other new classes of retrograde periodic orbits which are all very unstable. The orbits of these classes are presented in the figures:

- Fig. 18a, orbits surrounding all the bodies of the ring except the symmetric peripherals 3 and 6 . Figures $18 \mathrm{~b}$ and $18 \mathrm{c}$ show the characteristic and the stability curves respectively. The last curve has a minimum $a=1248$ at $C=0.45$, $x_{0}=-1.4919982$;

- Fig. 19a, these orbits curve at the peripheral 1 surrounding all the rest bodies. This class was found for negative values of $C$ (Fig. 19b). The stability curve (Fig. 19c) has a minimum $a=49.98$ at $C=-1.61\left(x_{0}=-2.0829631\right)$;

- Fig. 20a shows two orbits, curving at the peripheral 1 and surrounding only the symmetric peripherals 2 and 7 and the central body. The curve a $(C)$ (Fig. 20c) has a minimum $a=602$ at $C=1.82\left(x_{0}=-0.4635752\right)$. The characteristic curve of this class is shown in Fig. 20b. As $C$ decreases the orbits approach more and more the peripherals 2 and 7 and move away from peripheral 1 , while the curving smoothens. 

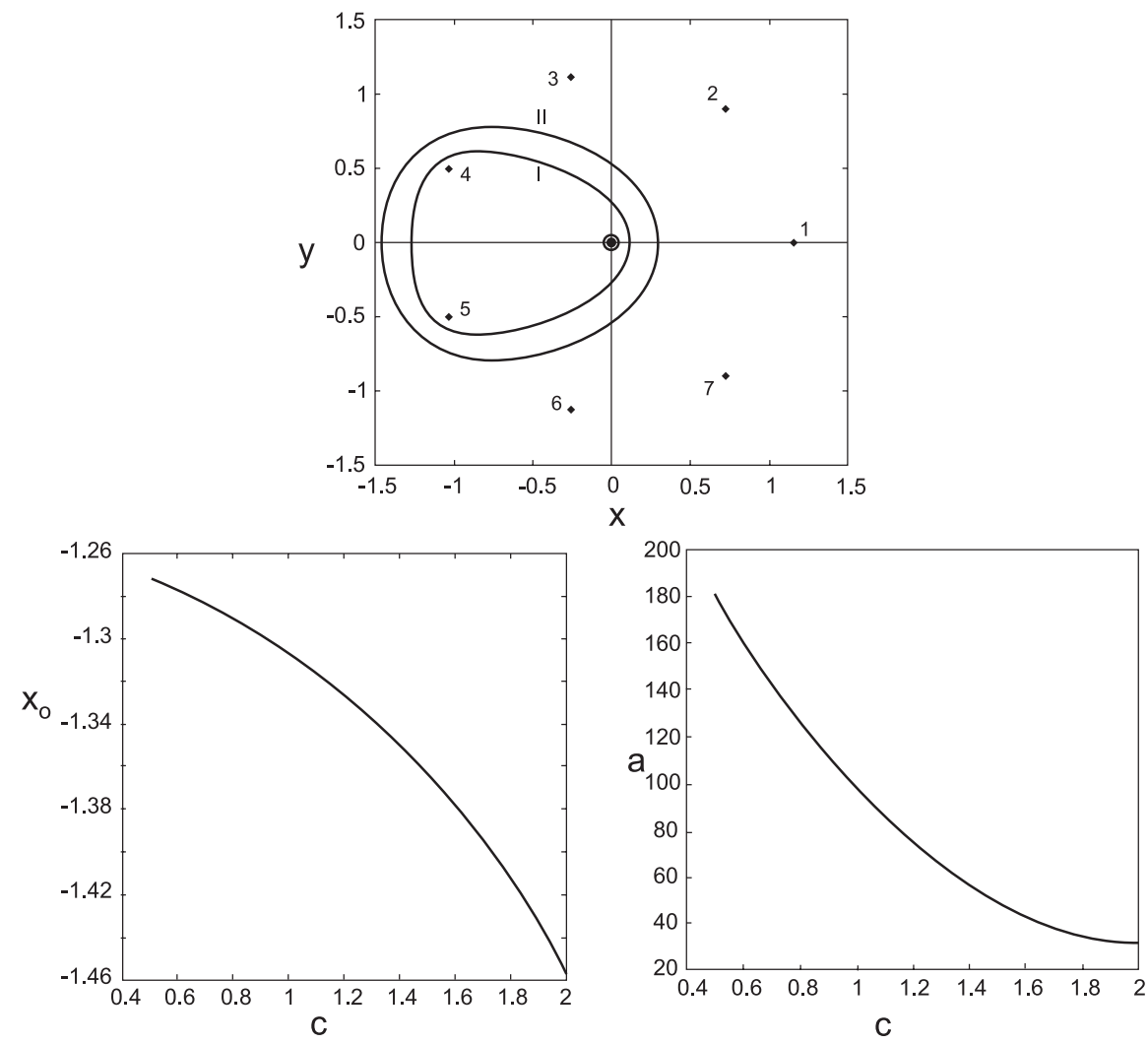

Fig. 17. a) Two simple periodic orbits, $N-1=7, \beta=2$ : for (I) $C=0.2, x_{0}=-1.2551793, a=252, T=1.372$ and (II) $C=2.2$, $x_{0}=-1.5120137, a=30.9, T=2.372$. b) Characteristic curve of the above class of the periodic orbits. $\left.\mathbf{c}\right)$ Stability curve of the above class of periodic orbits.
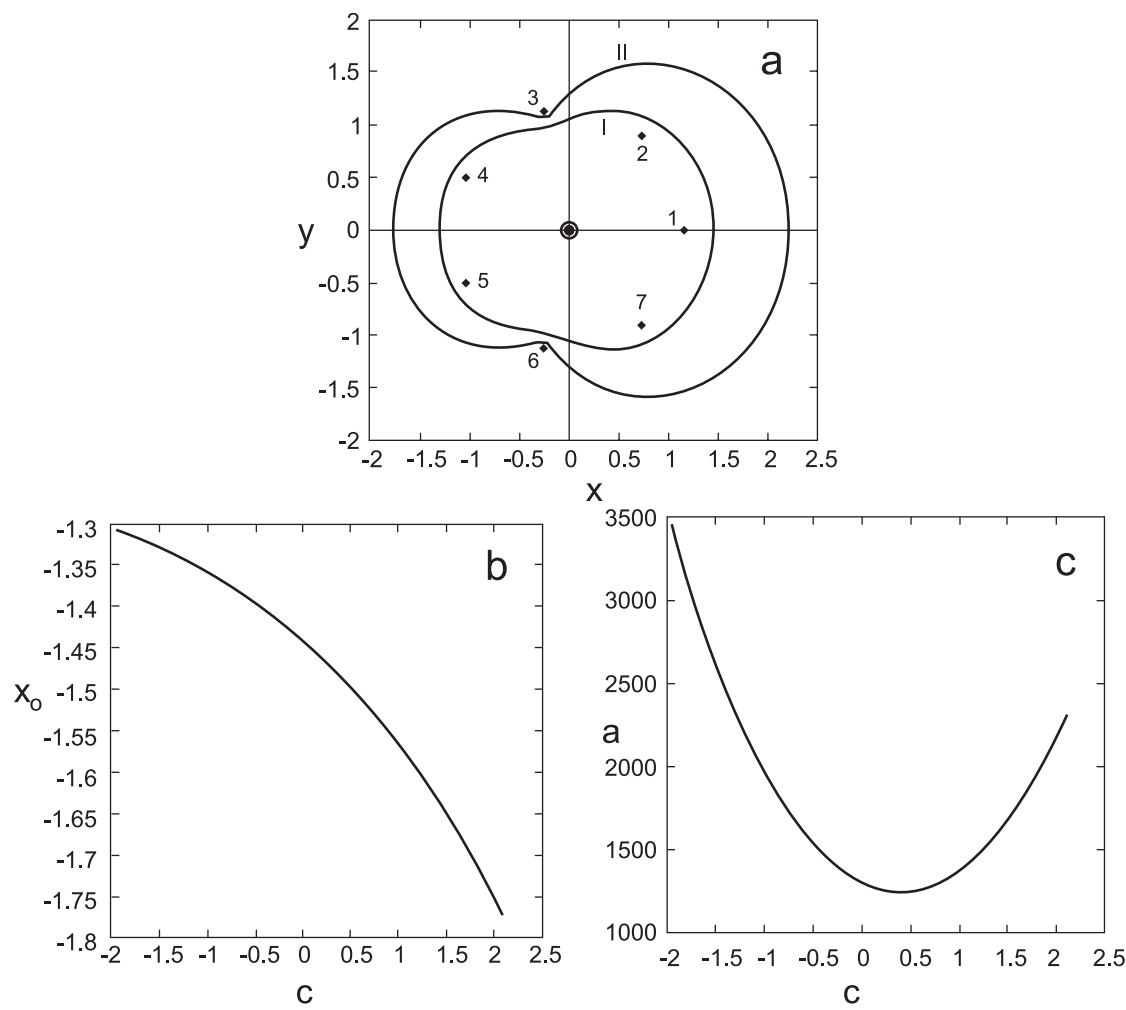

Fig. 18. a) Two simple periodic orbits, $N-1=7, \beta=2$ : for (I) $C=-2.2 x_{0}=-1.2988939, a=4077, T=2.467$ and (II) $C=2.2$, $x_{0}=-1.7944574, a=2451, T=4.977$. b) Characteristic curve of the above class of the periodic orbits. c) Stability curve of the above class of periodic orbits. 

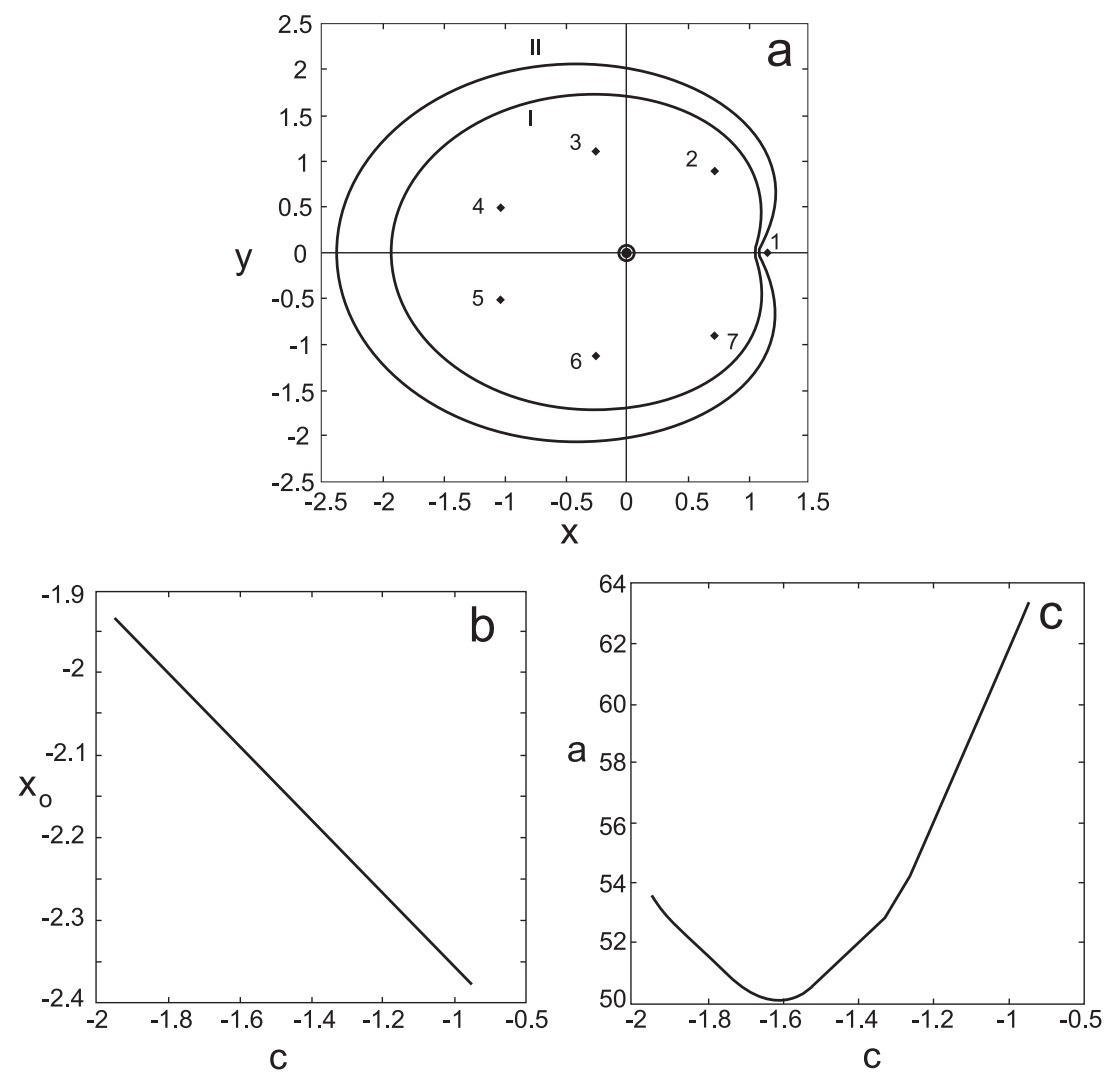

Fig. 19. a) Two simple periodic orbits, $N-1=7, \beta=2$ : for (I) $C=-2.1, x_{0}=-1.8732117, a=58.82, T=3.289$ and (II) $C=-0.8$, $x_{0}=-2.4392734, a=68.47, T=4.312$. b) Characteristic curve of the above class of the periodic orbits. c) Stability curve of the above class of periodic orbits.
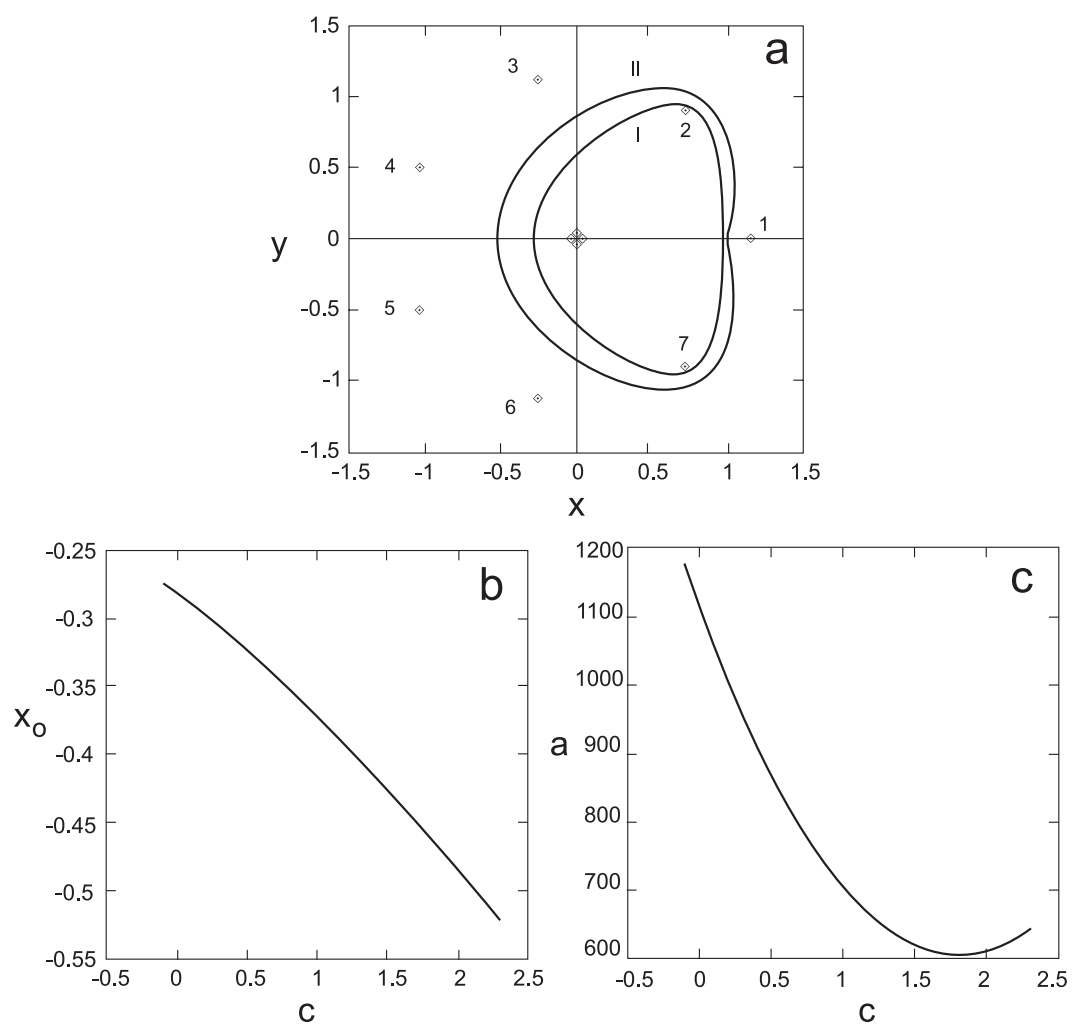

Fig. 20. a) Two simple periodic orbits, $N-1=7, \beta=2$ : for (I) $C=-0.2, x_{0}=-0.2682028, a=1242, T=1.684$, and (II) $C=2.3$, $x_{0}=-0.5206949, a=641, T=2.506$. b) Characteristic curve of the above class of the periodic orbits. c) Stability curve of the above class of periodic orbits. 

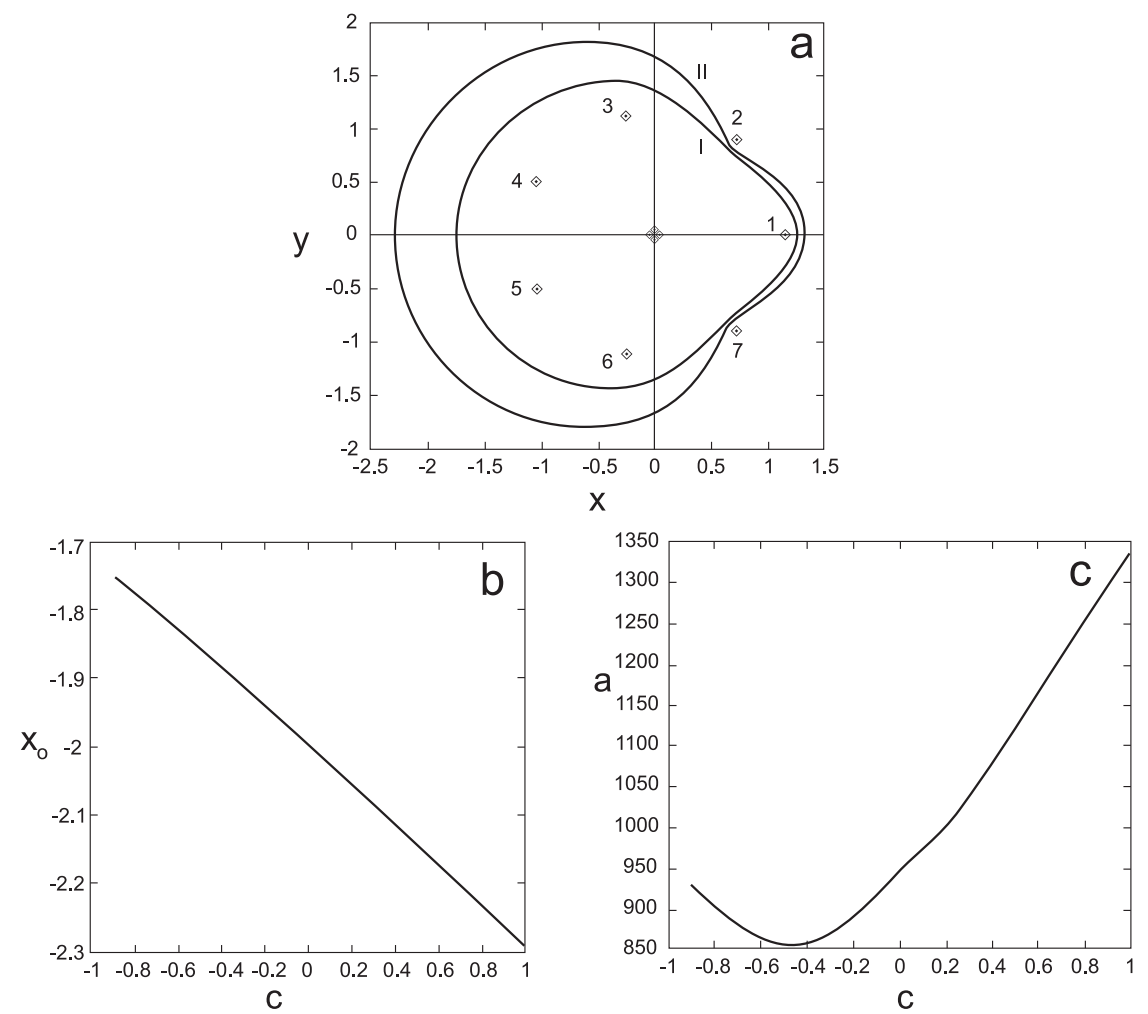

Fig. 21. a) Two simple periodic orbits, $N-1=7, \beta=2$ : for (I) $C=-1.0, x_{0}=-1.7286987, a=963, T=3.017$ and (II) $C=1.2$, $x_{0}=-2.3480647, a=1461$ and $T=4.372$. b) Characteristic curve of the above class of the periodic orbits. c) Stability curve of the above class of periodic orbits.
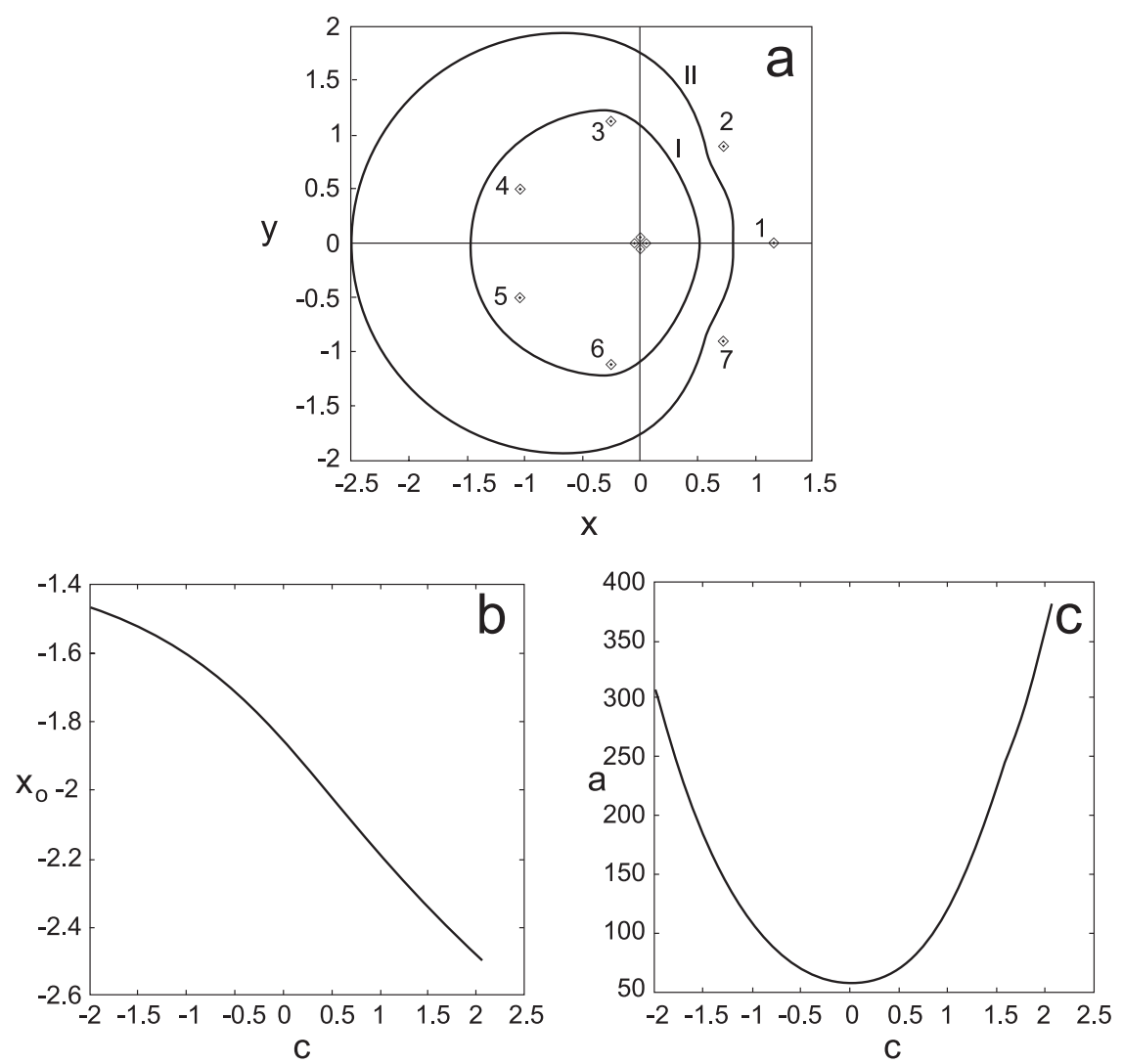

Fig. 22. a) Two simple periodic orbits, $N-1=7, \beta=2$ : for (I) $C=-2.2, x_{0}=-1.4459072, a=385, T=2.144$ and (II) $C=2.12$, $x_{0}=-2.5079447, a=410, T=4.688$. b) Characteristic curve of the above class of the periodic orbits. c) Stability curve of the above class of periodic orbits. 
We should note that the orbits 19a, 20a have a form similar to some of the classes $\mathrm{f}, \mathrm{b}$ of the restricted 3-body problem (Szebehely 1967; Pinotsis 1988);

- in Fig. 21a we give two orbits. The orbits of this class surround all the bodies except the peripherals 2 and 7. They present a behaviour similar to the previous orbits: that is, as $C$ decreases the two symmetric curves that exist at the peripherals 2 and 7 smoothen and the orbits move away from them, more and more approaching the peripherals 1, 3, 4, 5 and 6. The stability curve (Fig. 21c) has a minimum $a=857$ at $C=-0.44,\left(x_{0}=-1.8719401\right)$. Figure $21 \mathrm{~b}$ shows the characteristic curve of the class;

- Fig. 22a shows orbits of a form similar to the previous ones that do not surround the peripherals 1,2 and 7. As $C$ decreases they move nearer to the peripherals $3,4,5$ and 6 and farther away from the peripherals 1, 2 and 7. The two symmetric curves at the peripheral 2 and 7 become smoother. Also in Figs. 22b and 22c we show the characteristic and the stability curves respectively. The curve a $(C)$ which has a minimum $a=57.64$ at $C=-0.01\left(x_{0}=-1.8453618\right)$ shows the unstable character of this class.

\section{Concluding remarks}

We found the following main results.

We found four families of symmetric periodic orbits, first qualitatively and then numerically: two families of periodic orbits around the central body, one sidereally retrograde which is also synodically retrograde and another sidereally and synodically direct. Also, we found two families around all the peripherals, one retrograde in the rotating and fixed system and another direct in the fixed system and retrograde in the rotating system.

We did not numerically find a direct family of symmetric periodic orbits with respect to the rotating and the fixed system in the case $r>r_{\mathrm{p}}$, as we predicted theoretically.

In the case of the family $O_{\mathrm{R}}$ there is no family of double symmetric periodic orbits because there is no $1 \rightarrow 2$ bifurcation point (period doubling bifurcation) in this case (for $N-1=4,7$ and $\beta=2$ ). An open question to be investigated in the future is the existence of simple and double non-symmetric periodic orbits.

For the retrograde and direct periodic orbits around the central body: as the Jacobian constant increases, the orbits approach the central body and shrink to it $\left(C \rightarrow+\infty, x_{0} \rightarrow 0\right)$, while the stability coefficient $a \rightarrow 1$. As $C$ decreases the elliptical-shaped orbits go far from the central body, increasing in size. These two families of simple periodic orbits are stable in an interval $\left(C_{1}, C_{2}\right)$, and rings of stability develop in the inner region of the peripherals between $C_{1}<\infty, C_{2}=5.1357$ for the retrograde orbits and $C_{1}<\infty, C_{2}=7.30064$ for the direct orbits.

In the case of orbits around one peripheral there are also both retrograde and direct orbits, which are generated and evolve around one peripheral in the same way as the ones presented in Sect. 4.1.
We found that for $C>C_{\mathrm{cr}}\left(C_{\mathrm{cr}}\right.$ is a characteristic value for each of the two outer peripheral families) the stability index $a$ takes values very close to 1 , hence the orbits are critically stable. So, for $C \rightarrow \pm \infty$ the absolute value of $x_{0} \rightarrow \infty$ while the coefficient $a \rightarrow 1$, as we have theoretically predicted in Sect. 3 . These two families of simple periodic orbits are stable in an interval $\left(C_{1}, C_{2}\right)$, and rings of stability develop in the outer region of the peripherals between $C_{1}>-\infty, C_{2}=-4.20181$ for the retrograde orbits (family $O_{\mathrm{R}}$ ) and $C_{1}<+\infty, C_{2}=7.13$ for the family $O_{\mathrm{DR}}$.

In general, as the absolute value of $C$ decreases the periodic orbits of the four families approach the peripherals. After a characteristic value of $C$ near the distance $r_{\mathrm{p}}\left(|r|>r_{\mathrm{p}}\right)$ for each family, these families evolve in the $(x, y)$ plane. We thus obtain different classes of orbits which curve and twist among the peripherals. Orbits with $C$-negative result from orbits of the family $O_{\mathrm{R}}$, while orbits with $C$-positive result from orbits around the central body. An important characteristic of all these classes (that exist among the peripherals) is that they are strongly unstable having a minimum value for certain initial conditions. This is probably due to the gravitational effect of the peripherals to the small body's motion.

Also, the stability rings of retrograde periodic orbits are larger than those of the direct ones; thus, the retrograde orbits are more stable than the direct ones. This is very interesting for astronomical applications. So, the first three groups of families of planetary type orbits present important astronomical and astrophysical interest. The (retrograde) motion of a small body in a multi-body system is also of considerable interest. The $N$-body ring problem is a flexible system since the values of the $N-1$ and $\beta$ can change. Thus there are cases in Celestial Mechanics and in similar stellar formations that can be approached by a $N$-body ring problem. For example, the motion of a satellite in the asteroid belt and the motions of small bodies around planets or stars with rings.

The stability curves of the families of periodic orbits (retrograde and direct) have $1 \rightarrow 2$ bifurcation points. So, there are new bifurcating families of symmetric double periodic orbits with two branches in the form of a pitchfork. The bifurcating families are stable in the neighbourhood of the critical points. Also, there are other bifurcation points with $|a+d| / 2=1$. These points are critical points of type 4 . Thus, here there are bifurcations with new families of non-symmetric simple periodic orbits of the same period.

In the case $N-1=4, \beta=2$ the behaviour and the evolution of the periodic orbits with $\mathrm{C}$ is similar to the previous $N-1=7, \beta=2$ case. We found families of both stable and unstable retrograde and direct periodic orbits around the central body, around all the peripherals as well as among them. In a future work we will study the change of the size of the stability rings for different values of $N-1$ and $\beta$ as well as the evolution of the different types of bifurcations. 
Acknowledgements. I thank Prof. G. Contopoulos for his useful comments. I also thank the anonymous referee for detailed comments.

\section{References}

Contopoulos, G. 1970, AJ, 75, 96

Contopoulos, G. 2002, Order and Chaos in Dynamical Astronomy (New York: Springer Verlag)

Hadjidemetriou, J. 1977, Cel. Mech., 16, 61

Hadjidemetriou, J. 1979, Stability of periodic planetary type orbits of the general planar $N$-body problem, in Dynamics of the Solar system, ed. R. I. Duncombe, IAU, 23

Hadjidemetriou, J. 1989, Periodic Orbits and Stability, Programme Univer. Europ. Erasmus, Thessaloniki

Hénon, M. 1965, Ann. Astrophys., 28, 992

Hénon, M. 1970, in Periodic orbits, Stability and Resonances, ed. G. Giacaglia (Holand: D. Reidel), 349

Deprit, A., \& Henrard, J. 1968, Adv. Astron. Astrophys., 6, 1
Kalvouridis, T. J. 1999a, Ap\&SS, 260, 309

Kalvouridis, T. J. 1999b, Ap\&SS, 266, 467

Markellos, V. V., Papadakis, K. E., \& Perdios, E. A. 1997, The plane restricted five-body problem, in New Trends in Astronomy and Astrophysics, 6th European Astronomical Meeting (JENAM 97), Thessaloniki, Greece

Meyer, G. 1981, Cel. Mech., 23, 69

Palmore, J. 1982, Cel. Mech., 28, 17

Pinotsis, A. D. 1987, A\&A, 174, 317

Pinotsis, A. D. 1988, in Long Term Dynamical Behaviour of Natural and Artificial $N$-Body Systems, ed. A. E. Roy, 465 (Kluwer Academic Publishers)

Pinotsis, A. D., Tsironis, C., \& Kalvouridis, T. J. 2002, Symmetric periodic motion in a ring problem; in 5th Hellenic Astronomical Conference, Proceedings, ed. D. Hatzidimitriou, et al., Fodele, Crete

Poincaré, H. 1899, Les méthodes nouvelles de la mécanique céleste (Paris: Gauthier Villars)

Szebehely, V. 1967, Theory of orbits (New York: Academic Press) 